\title{
Stresses in a Plate Uniformly Compressed Over Portions of Its Two Opposite Edges
}

\author{
Michael Chi* and Wilhelmina D. Kroll
}

(April 30, 1962)

\begin{abstract}
The stress distributions in rectangular plates with symmetrical uniform loads over central portions of two opposite edges are evaluated theoretically. Results are shown to be applicable to the stress analysis of a uniaxially loaded plate which is elastically constrained by an unloaded plate perpendicular to it. This structural configuration is often found in welded assemblies.

A comparison of experimental results with theoretical results for a simple welded structure shows reasonable agreement.
\end{abstract}

\section{Introduction}

Following the occurrence of serious structural failures in welded ships during World War II, investigations were undertaken to determine the cause and possible remedies for these failures. It was apparent to early investigators that at least part of the failures in large monolithic structures could be attributed to the brittle behavior of material commonly considered ductile. Experimental studies $[1,2]^{1}$ were conducted to confirm this assumption. The specimens used in these studies, as shown in figure 1 which is taken from reference [1], represented interrupted bulkhead intersections in welded tankers. Information from these and other studies led to modifications of existing ships and improved design of new vessels, and resulted in a substantially lower number of failures [3].

Strain measurements in these specimens also revealed the existence of a complex stress distribution near the intersections of two plates in the test specimens. No theoretical analysis was found to explain the distribution of stresses in the test specimens. This may be due to the fact that a rigorous investigation of even a simple monolithic structure, such as shown in figure 2 , in which the base plate under uniaxial tension is elastically constrained by two flanges, is a three-dimensional problem with mixed boundary conditions and is difficult to solve.

However, it is shown herein that the problem of determining the stress distribution in the structure of figure 2, consisting of plates whose thickness is small compared to other dimensions, can be reduced to the stress problem of a plate loaded over portions of its boundaries. The latter problem has been adequately treated in standard texts but numerical results are available only for the cases of narrow strips under concentrated loads $[4,5]$. This paper gives the numerical results of stresses due to a uniform load over central portions of two opposite edges of plates of

*Present address: The Catholic University of America, Washington, D.C.

1 Figures in brackets indicate the literature references on page 346. various dimensions and indicates how the results may be used to estimate the stress distribution near the intersection of two perpendicular thin plates of arbitrary lengths.

\section{Analysis}

The simplified welded structure shown in figure 2 can be considered to be equivalent to the plate shown in figure 3 since, in these figures, the shaded portions which correspond to each other have the same restraining effect on the respective base plates. The equivalence of these two problems can be verified as follows. If the plate shown in figure 3 is thin, the shaded portions at the ends can be bent through 90 degrees without changing the stress picture. Then the plate can be cut at the middle along the $y$-axis. The continuity effect is replaced by boundary forces which are equal to $p$. The two halves of the plate may then be joined at the bent ends with the shaded portions in the up and down positions shown in figure 2. Note that the deformations along the bent edges are compatible because of symmetry and that the loads $p$ cancel each other when the halves of the plate are joined. The boundary forces produced by cutting. then become the end forces shown in figure 2 ; hence these two figures are equivalent to each other.

The analysis of the plate in figure 3 , therefore, will form the basis of evaluating the stresses in the welded structure of figure 2.

A direct solution of the problem shown in figure 3 is rather difficult. However, the problem becomes almost trivial through the use of the superposition principle. It will be shown in the next few paragraphs that the problem shown in figure 3 is statically equivalent to the combination of cases shown in figure $4 \mathrm{~b}$ and $4 \mathrm{c}$.

First, it is assumed that the joints between the shaded and unshaded portions of the plate shown in figure 3 are cut. A uniform tensile load would produce rigid body displacements of the shaded portions of the plate without producing stresses in them. 

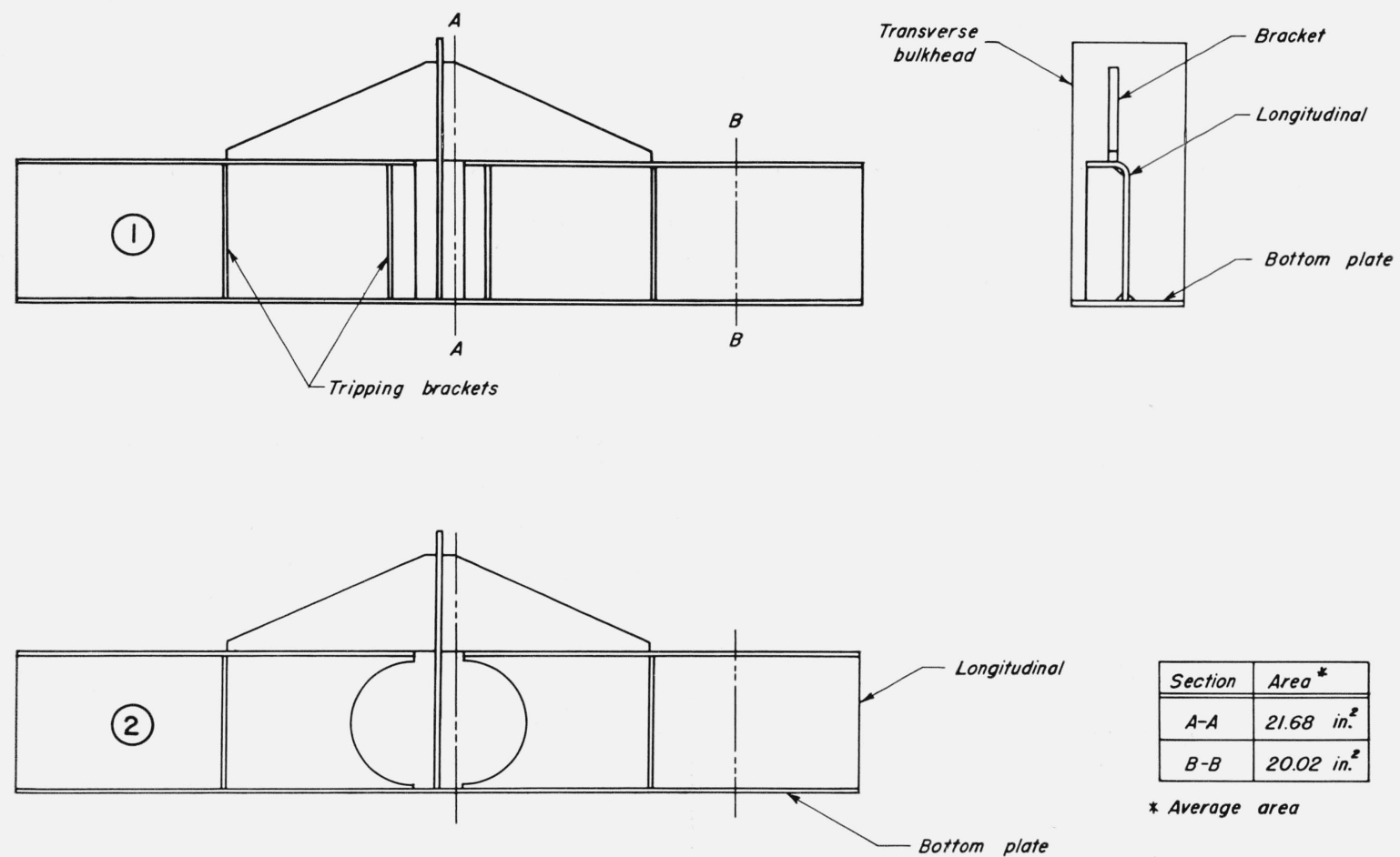

\begin{tabular}{|c|l|}
\hline Section & Area $^{*}$ \\
\hline \hline$A-A$ & 21.68 in. $^{2}$ \\
\hline$B-B$ & 20.02 in. $^{2}$ \\
\hline
\end{tabular}

* Average area
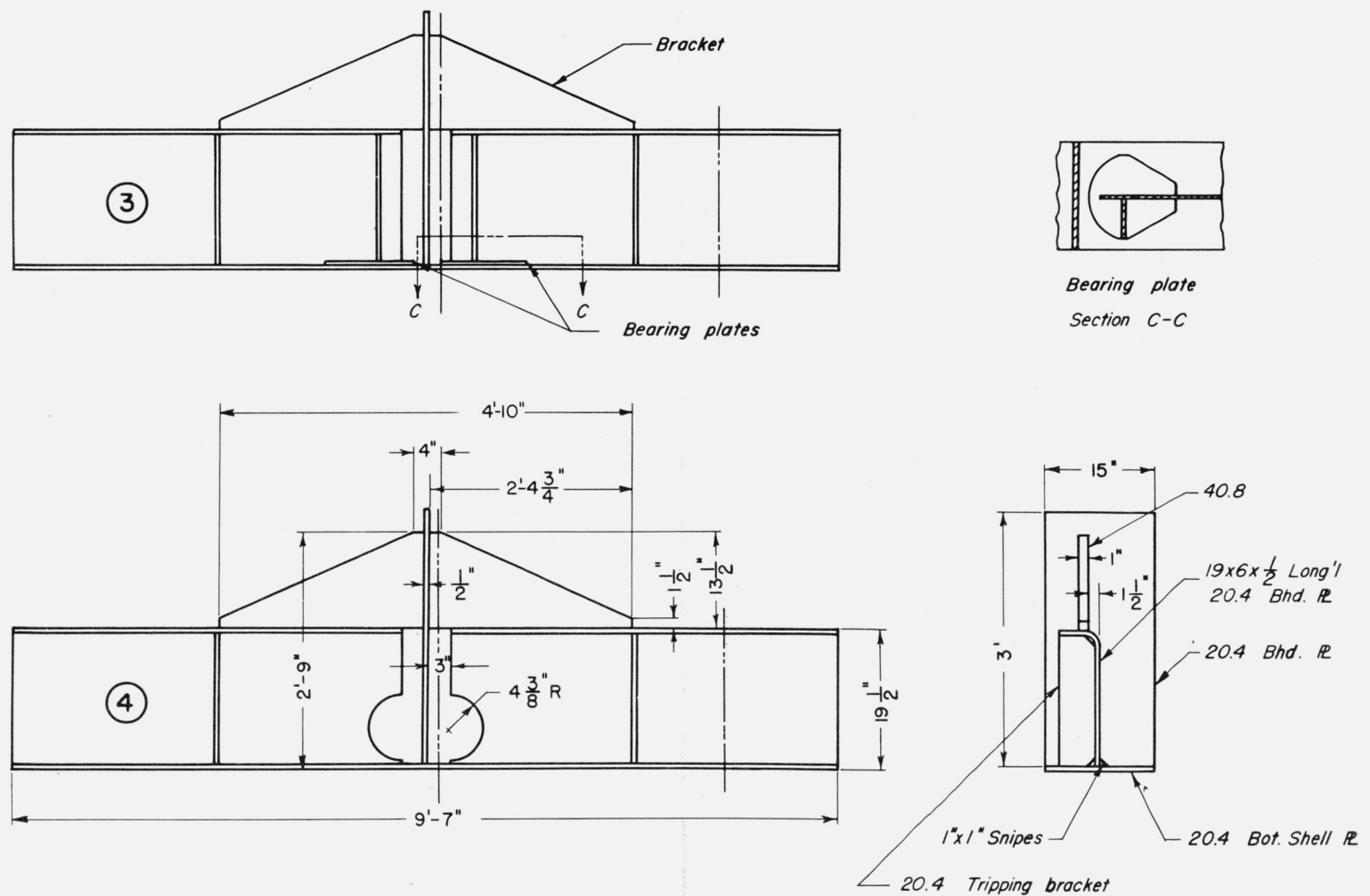

Figure 1. Dimensions and details of interrupted longitudinal specimens. Figure 1 of reference [1]. 
For the central part of the plate of figure 3, which is under the unit tensile load $p$, the stresses and strains in the transverse direction of the plate would be:

and

$$
\sigma_{y}^{\prime}=0
$$

$$
\epsilon_{y}=-\nu \frac{p}{E}
$$

where

$\sigma_{y}^{\prime}=$ stress in $y$-direction

$\boldsymbol{\epsilon}_{y}=$ strain in $y$-direction

$p=$ load per unit area

$E=$ Young's modulus

$\nu=$ Poisson's ratio.

The deformed configuration of the plate is shown in figure $4 \mathrm{a}$.

To prevent the contraction corresponding to $\epsilon_{y}$, a uniformly distributed load $q$ must act along the edges of the central portion of the plate. See figure $4 \mathrm{~b}$. The magnitude of $q$ is then,

$$
q=-E \boldsymbol{\epsilon}_{y}=\nu p .
$$

Due to the uniform load $q$, the deformations in the shaded and unshaded portions of the plate are compatible. The portions of the plate can now be joined to restore continuity. However, to be consistent with the condition of a free boundary, a load $-q$ must be applied to the edges of the central portion of the plate to neutralize the edge load $q$ as shown in figure 4c. It is clear that the effect of loads $p$ on the structure shown in figure 3 is equivalent to the effect of a system of forces shown in figure $4 \mathrm{~b}$ superimposed on another system of forces shown in figure $4 \mathrm{c}$.

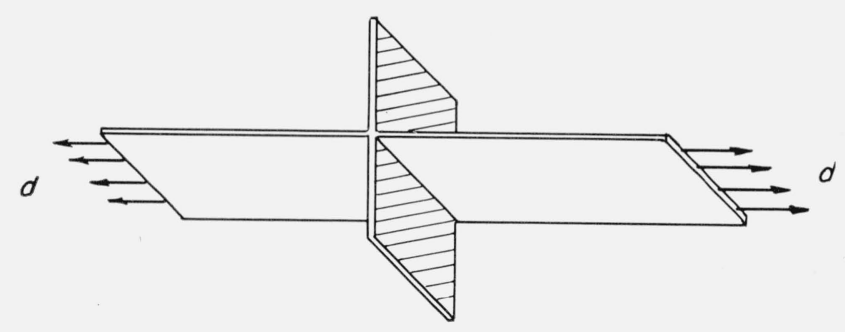

FIGURE 2. Simple welded structure.

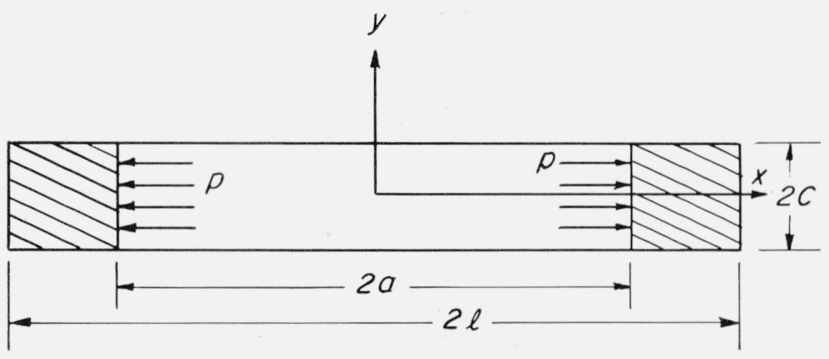

FiguRE 3. Structure considered equivalent to welded structure.

\section{Theory}

In order to determine the stresses in a plate such as shown in figure 3 , use has been made of existing theoretical work. The expressions for the stresses in a plate, figure 5, with a symmetrical load distribution on two of the boundaries representable by a Fourier cosine series have been developed in texts on elasticity. See, for example, [6].
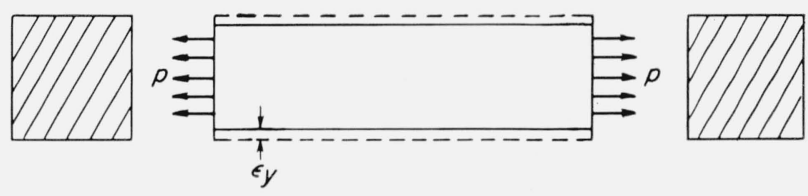

(a) Contraction of bose plate under tensile forces

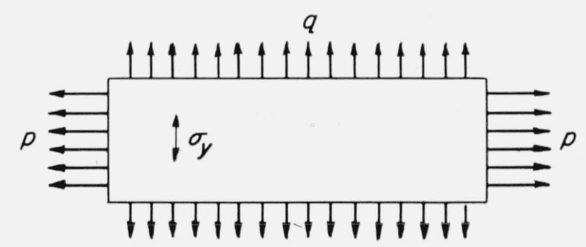

(b) Uniform lood added to prevent contraction of part of plate under tensile forces

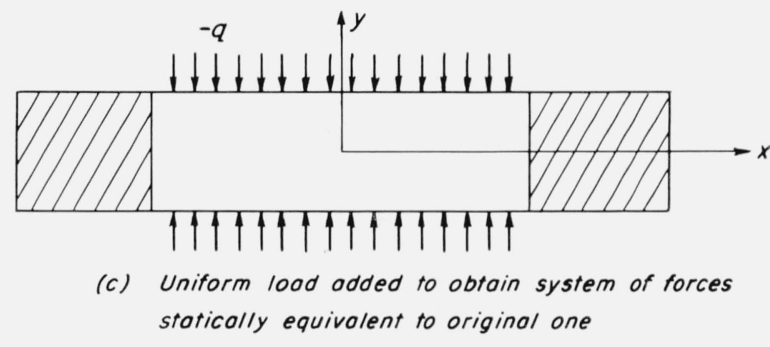

Figure 4. Method of analyzing welded joint.

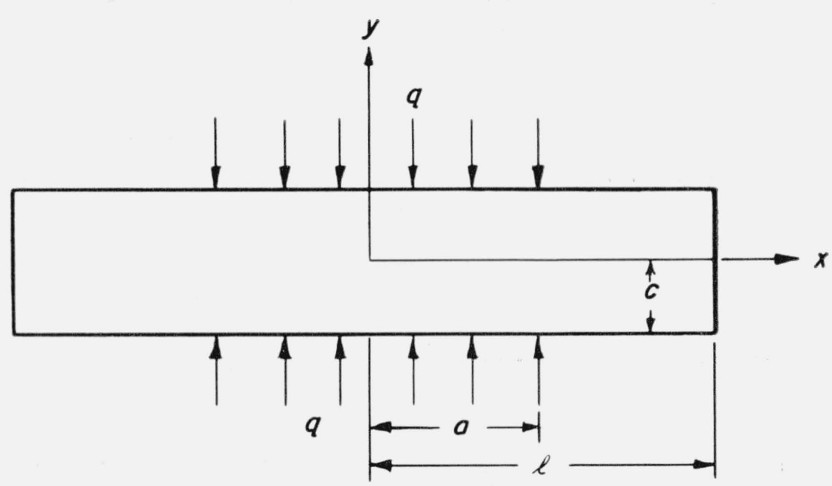

Figure 5. Dimensions of plate under uniform load q. 
The equations for the stresses are:

$$
\begin{aligned}
\sigma_{x} & =\frac{A_{0}}{2}+\sum_{m=1}^{\infty} 2 A_{m} \frac{\left(\frac{m \pi c}{l} \cosh \frac{m \pi c}{l}-\sinh \frac{m \pi c}{l}\right) \cosh \frac{m \pi y}{l}-\frac{m \pi y}{l} \sinh \frac{m \pi y}{l} \sinh \frac{m \pi c}{l}}{\sinh 2 \frac{m \pi c}{l}+2 \frac{m \pi c}{l}} \cos \frac{m \pi x}{l} \\
\sigma_{y} & =-\frac{A_{0}}{2}-\sum_{m=1}^{\infty} 2 A_{m} \frac{\left(\frac{m \pi c}{l} \cosh \frac{m \pi c}{l}+\sinh \frac{m \pi c}{l}\right) \cosh \frac{m \pi y}{l}-\frac{m \pi y}{l} \sinh \frac{m \pi y}{l} \sinh \frac{m \pi c}{l}}{\sinh 2 \frac{m \pi c}{l}+2 \frac{m \pi c}{l}} \cos \frac{m \pi x}{l} \\
\tau_{x y} & =\frac{A_{0}}{2}+\sum_{m=1}^{\infty} 2 A_{m} \frac{\frac{m \pi c}{l} \cosh \frac{m \pi c}{l} \sinh \frac{m \pi y}{l}-\frac{m \pi y}{l} \cosh \frac{m \pi y}{l} \sinh \frac{m \pi c}{l}}{\sinh 2 \frac{m \pi c}{l}+2 \frac{m \pi c}{l}} \sin \frac{m \pi x}{l}
\end{aligned}
$$

where

$$
\begin{aligned}
& a=\text { half length of loaded portion of plate; } \\
& c=\text { half width of plate; } \\
& l=\text { half length of plate; } \\
& x, y=\text { coordinates of points in plate, meas- } \\
& \text { ured from the mid-length and } \\
& \text { mid-width of the plate, respec- } \\
& \text { tively; }
\end{aligned}
$$

$A_{0}, A_{1}$, etc. $=$ Fourier coefficients.

If a normal load $q$ is uniformly distributed over the central portion 2a of the plate, the Fourier coefficients for $m=1,2,3 \ldots \infty$ are [7]:

$$
\begin{aligned}
A_{m} & =\frac{1}{l} \int_{-a}^{a} q \cos \frac{m \pi x}{l} d x \\
& =\frac{1}{l} \frac{l}{m \pi} \int_{-a}^{a} q \frac{m \pi}{l} \cos \frac{m \pi x}{l} d x \\
& \left.=\frac{q}{m \pi} \sin \frac{m \pi x}{l}\right]_{-a}^{a} \\
& =\frac{2 q}{m \pi} \sin \frac{m \pi a}{l} .
\end{aligned}
$$

The coefficient

$$
A_{0}=\frac{1}{l} \int_{-a}^{a} q d x
$$

For $\sigma_{x}$,

$$
\begin{aligned}
A_{0} & =\frac{1}{l} \int_{-a}^{a} 0 d x \\
& =0 .
\end{aligned}
$$

For $\sigma_{y}$,

$$
\begin{aligned}
A_{0} & =\frac{1}{l} \int_{-a}^{a} q d x \\
& =\frac{2 a q}{l} .
\end{aligned}
$$

For $\tau_{x y}$

$$
\begin{aligned}
A_{0} & =\frac{1}{l} \int_{-a}^{a} 0 d x \\
& =0 .
\end{aligned}
$$

Substituting the values of the coefficients and the equivalent expression $2 \sinh \frac{m \pi c}{l} \cosh \frac{m \pi c}{l}$ for $\sinh 2 \frac{m \pi c}{l}$, the equations for the stresses become:

$$
\begin{aligned}
& \sigma_{x}=\sum_{m=1}^{\infty} \frac{2 q}{m \pi} \sin \frac{m \pi a}{l} \cos \frac{m \pi x}{l}\left\{\frac{\left(\frac{m \pi c}{l} \cosh \frac{m \pi c}{l}-\sinh \frac{m \pi c}{l}\right) \cosh \frac{m \pi y}{l}-\frac{m \pi y}{l} \sinh \frac{m \pi c}{l} \sinh \frac{m \pi y}{l}}{\sinh \frac{m \pi c}{l} \cosh \frac{m \pi c}{l}+\frac{m \pi c}{l}}\right\} \\
& \sigma_{y}=-\frac{q a}{l}-\sum_{m=1}^{\infty} \frac{2 q}{m \pi} \sin \frac{m \pi a}{l} \cos \frac{m \pi x}{l}\left\{\frac{\left(\frac{m \pi c}{l} \cosh \frac{m \pi c}{l}+\sinh \frac{m \pi c}{l}\right) \cosh \frac{m \pi y}{l}-\frac{m \pi y}{l} \sinh \frac{m \pi c}{l} \sinh \frac{m \pi y}{l}}{\sinh \frac{m \pi c}{l} \cosh \frac{m \pi c}{l}+\frac{m \pi c}{l}}\right\} \\
& \tau_{x y}=\sum_{m=1}^{\infty} \frac{2 q}{m \pi} \sin \frac{m \pi a}{l} \sin \frac{m \pi x}{l}\left\{\frac{\frac{m \pi c}{l} \cosh \frac{m \pi c}{l} \sinh \frac{m \pi y}{l}-\frac{m \pi y}{l} \sinh \frac{m \pi c}{l} \cosh \frac{m \pi y}{l}}{\sinh \frac{m \pi c}{l} \cosh \frac{m \pi c}{l}+\frac{m \pi c}{l}}\right\} .
\end{aligned}
$$

The above equations were used in computing the stresses in plates for various dimensions and loadings unless modified as indicated in the following sections. 


\section{Computations}

The computations for the stresses in plates with discontinuous loadings were carried out in an IBM 704 electronic calculator. The dimensions of the plates and the parts of the plates under uniform load are given in table 1 .

The values of the stresses using eq (4) converge rather slowly; a large number of terms are usually necessary to assure sufficient accuracy in the results. Based on hand computation for the stresses at particular points in the plate, the following procedure was used to obtain stresses to five decimal places. At first, the stresses were computed for values of $m$ for which $0<\frac{m \pi a}{l}<2 \pi$; then the stresses were computed for values of $m$ for which $0<\frac{m \pi a}{l}<4 \pi$ and so on. Each time the angle $\frac{m \pi a}{l}$ increased by $2 \pi$, a comparison was made between the value of $\sigma_{x}$ for this cycle and the preceding one. If the value of $\sigma_{x}$ changed by 0.00001 or less, the computation was stopped. It was thought that this criterion was adequate but in several instances the computation was stopped before the series had converged. Therefore except on the boundary, 100 terms in the series were used for all points, as long as $\frac{m \pi(c-y)}{l}<16$. The computation was stopped if the quantity $\frac{m \pi(c-y)}{l}$ became greater than 16 since the increment in the stresses would be essentially zero. For angles $\frac{m \pi c}{l}$ or $\frac{m \pi y}{l}$ greater than 8 , the hyperbolic sine was considered to be equal to the hyperbolic cosine with no detectable error within the limits of accuracy desired. The terms under the summation sign in eq (4) were accordingly modified as the summing proceeded to avoid overflow in the computer when computing the hyperbolic functions of very large angles.

Along the boundaries, $y= \pm c$, the values of the stresses showed no signs of convergence, even when as many as 300 terms were taken, but became oscillatory. The equation for $\sigma_{y}$ at $y=c$ is from eq (4):

$$
\left.\sigma_{y}\right|_{y=c}=-\frac{q a}{l}-\sum_{m=1}^{\infty}\left\{\frac{2 q}{m \pi} \sin \frac{m \pi a}{l} \cos \frac{m \pi x}{l}\right\}\left\{\frac{\frac{m \pi c}{l} \cosh ^{2} \frac{m \pi c}{l}+\sinh \frac{m \pi c}{l} \cosh \frac{m \pi c}{l}-\frac{m \pi c}{l} \sinh ^{2} \frac{m \pi c}{l}}{\sinh \frac{m \pi c}{l} \cosh \frac{m \pi c}{l}+\frac{m \pi c}{l}}\right\}
$$

TABLE 1. Dimensions of plates investigated and computed maximum and minimum stresses

\begin{tabular}{|c|c|c|c|c|c|c|c|c|c|}
\hline 1 & 2 & 3 & 4 & 5 & 6 & 7 & 8 & 9 & 10 \\
\hline \multirow{2}{*}{ Case } & \multirow{2}{*}{$\begin{array}{c}\text { Loaded } \\
\text { length } \\
\text { of plate, } \\
2 a\end{array}$} & \multirow{2}{*}{$\begin{array}{l}\text { Nonloaded } \\
\text { length of } \\
\text { plate, } \\
2(l-a)\end{array}$} & \multirow{2}{*}{$\begin{array}{l}\text { Width of } \\
\text { plate } \\
2 c\end{array}$} & Loaded length & Loaded length & Nonloaded length & \multicolumn{3}{|c|}{$\begin{array}{l}\text { Maximum and minimum } \\
\text { stresses }\end{array}$} \\
\hline & & & & $\begin{array}{l}\text { Total length } \\
\frac{a}{l}\end{array}$ & $\begin{array}{l}\text { Width } \\
\frac{a}{c}\end{array}$ & $\frac{\text { Width }}{\frac{l-a}{c}}$ & $\sigma_{x} / q$ & $\sigma_{y} / q$ & $\tau_{x y} / q$ \\
\hline 1 & 100 & 40 & 20 & 0.71 & 5. 0 & 2.0 & \pm 0.500 & $\begin{array}{r}0.040 \\
-1.025\end{array}$ & $\begin{array}{r}0.232 \\
-.040\end{array}$ \\
\hline 2 & 100 & 30 & 20 & .77 & 5. 0 & 1.5 & \pm .500 & $\begin{array}{r}0.099 \\
-1.023\end{array}$ & $\begin{array}{r}.230 \\
-.049\end{array}$ \\
\hline 3 & 100 & 20 & 20 & .83 & 5. 0 & 1.0 & $\begin{array}{r}.457 \\
-.543\end{array}$ & $\begin{array}{r}0.176 \\
-1.023\end{array}$ & $\begin{array}{r}.260 \\
-.047\end{array}$ \\
\hline 4 & 100 & 10 & 20 & .91 & 5. 0 & 0.5 & $\begin{array}{r}.265 \\
-.735\end{array}$ & $\begin{array}{r}0.126 \\
-1.038\end{array}$ & $\begin{array}{r}.180 \\
-.059\end{array}$ \\
\hline 5 & 40 & 100 & 20 & .29 & 2. 0 & 5. 0 & \pm .500 & $\begin{array}{r}0.023 \\
-1.025\end{array}$ & $\begin{array}{r}.269 \\
-.038\end{array}$ \\
\hline 6 & 30 & 110 & 20 & .21 & 1.5 & 5.5 & \pm .500 & $\begin{array}{r}0.022 \\
-1.044\end{array}$ & $\begin{array}{r}.269 \\
-.038\end{array}$ \\
\hline 7 & 20 & 120 & 20 & .14 & 1. 0 & 6. 0 & $\begin{array}{r}.492 \\
-.508\end{array}$ & $\begin{array}{r}0.022 \\
-1.024\end{array}$ & $\begin{array}{r}.272 \\
-.038\end{array}$ \\
\hline 8 & 10 & 130 & 20 & .07 & 0.5 & 6.5 & $\begin{array}{r}.387 \\
-.613\end{array}$ & $\begin{array}{r}0.018 \\
-1.001\end{array}$ & $\begin{array}{r}.288 \\
-.030\end{array}$ \\
\hline
\end{tabular}

This reduces to

$$
\left.\sigma_{y}\right|_{y=c}=-\frac{q a}{l}-\sum_{m=1}^{\infty} \frac{2 q}{m \pi} \sin \frac{m \pi a}{l} \cos \frac{m \pi x}{l} .
$$

However, from the loading shown on figure 5,

$$
\left.\sigma_{y}\right|_{y=c}=-q \text { for } 0 \leqq x \leqq a
$$

and

$$
\left.\sigma_{y}\right|_{y=c}=0 \text { for } a \leqq x \leqq l .
$$


When the stresses in the $y$-direction are known, for example, and those in the $x$-direction are expressed by a slowly convergent series, the stresses $\sigma_{x}$ at the boundary can be computed by making use of the following relationship [8].

$$
\left.\sigma_{x}\right|_{y=c}=\left[\left.\sigma_{x}\right|_{y=c}-\left.\sigma_{y}\right|_{y=c}\right]+\left.\sigma_{y}\right|_{y=c} .
$$

The equations for $\sigma_{x}$ and $\sigma_{y}$, eq (4), substituted into eq (8) give

$$
\left.\sigma_{x}\right|_{y=c}=\frac{q a}{l}+\sum_{m=1}^{\infty} \frac{4 q c}{l} \frac{\sin \frac{m \pi a}{l} \cos \frac{m \pi x}{l}}{\sinh \frac{m \pi c}{l} \cosh \frac{m \pi c}{l}+\frac{m \pi c}{l}}+\left.\sigma_{y}\right|_{y=c} .
$$

The last term is given by eq (7). The series part of eq (9) is rapidly convergent and only 20 terms were needed in the summation as long as the value of $\frac{m \pi c}{l}$ was less than 9 . When the value of $\frac{m \pi c}{l}$ became greater than 9 , the computation was stopped as any error in not taking more terms in the series would be insignificant.

\section{Correction of Boundary Values}

An inspection of the computed values for cases 1 to 4 showed that eq (4) satisfied the boundary condition that the shearing stresses at the boundary $x= \pm l$ be zero but did not satisfy the condition that the stresses normal to the boundary be zero. The stress residuals in the $x$-direction were small for case 1 but became progressively larger as the extent of the loading approached the boundary of the plate.

For cases 1 and 2 , table 1 , the distribution of the stress residuals followed approximately a cosine curve (see the solid curve on fig. 6a and 6b). For case 3, the distribution of the stress residuals approximated a parabola, figure $6 \mathrm{c}$, and for case 4 , a combination of a parabola and cosine curve, figure $6 \mathrm{~d}$. For cases 5 to 8 where the loading is on a relatively small area and distant from the plate boundary, there were no stress residuals in the $x$-direction.

To eliminate the stresses $\sigma_{x}$ at $x= \pm l$ for cases 1 and 2 and so satisfy the boundary conditions, stresses were computed by use of the following equations [9], and added to those previously computed

$$
\begin{aligned}
& \sigma_{x}=-2 A \frac{\left(\frac{\pi l}{c} \cosh \frac{\pi l}{c}+\sinh \frac{\pi l}{c}\right) \cosh \frac{\pi x}{c}-\frac{\pi x}{c} \sinh \frac{\pi x}{c} \sinh \frac{\pi l}{c} \cos \frac{\pi y}{c}}{\sinh \frac{2 \pi l}{c}+2 \frac{\pi l}{c}} \\
& \sigma_{y}=2 A \frac{\left(\frac{\pi l}{c} \cosh \frac{\pi l}{c}-\sinh \frac{\pi l}{c}\right) \cosh \frac{\pi x}{c}-\frac{\pi x}{c} \sinh \frac{\pi l}{c} \sinh \frac{\pi x}{c}}{\sinh \frac{2 \pi l}{c}+2 \frac{\pi l}{c}} \cos \frac{\pi y}{c} \\
& \tau_{x y}=2 A \frac{\frac{\pi l}{c} \cosh \frac{\pi l}{c} \sinh \frac{\pi x}{c}-\frac{\pi x}{c} \sinh \frac{\pi l}{c} \cosh \frac{\pi x}{c}}{\sinh \frac{2 \pi l}{c}+\frac{2 \pi l}{c} \sin \frac{\pi y}{c},}
\end{aligned}
$$

where $A=-0.017 q$ and $-0.055 q$ for cases 1 and 2 , respectively.

The stress residuals for case 3 approximated a parabola, figure 6c. To satisfy the boundary condition that the stresses in the $x$-direction be zero at the boundaries $x= \pm l$, the approximate solution for the stresses when the forces on the ends are distributed according to a parabolic law [10] was used. If, as it is pointed out, the stress function is in the form of

$$
\begin{array}{r}
\phi=\frac{1}{2} P y^{2}\left(1-\frac{1}{6} \frac{y^{2}}{c^{2}}\right)+\left(x^{2}-a^{2}\right)^{2}\left(y^{2}-c^{2}\right)^{2} \\
\left(\alpha_{1}+\alpha_{2} x^{2}+\alpha_{3} y^{2}\right)
\end{array}
$$

and the plate is twice as long as it is wide, the stress distribution across the plate at $x=0$ is almost uniform. The equations derived for the stresses with this stress function were used, therefore, to compute the corrections to the stresses. The equations are:

$$
\begin{array}{r}
\sigma_{x} / q=0.374\left(1-\frac{y^{2}}{c^{2}}\right)+4\left(\frac{x^{2}}{a^{2}}-1\right)^{2}\left(3 \frac{y^{2}}{c^{2}}-1\right)(0.02986 \\
\left.+0.04675 \frac{x^{2}}{a^{2}}+0.00683 \frac{y^{2}}{c^{2}}\right)-0.2455 \\
\sigma_{y} / q=4\left(\frac{y^{2}}{c^{2}}-1\right)^{2}\left(3 \frac{x^{2}}{a^{2}}-1\right)\left(\frac{c^{2}}{a^{2}}\right)\left(0.02986+0.04675 \frac{x^{2}}{a^{2}}\right. \\
\left.+0.00683 \frac{y^{2}}{c^{2}}\right)+0.0935\left(\frac{y^{2}}{c^{2}}-1\right)^{2} \\
\left(\frac{x^{2}}{a^{2}}-1\right)\left(\frac{c^{2}}{a^{2}}\right)\left(9 \frac{x^{2}}{a^{2}}-1\right)
\end{array}
$$



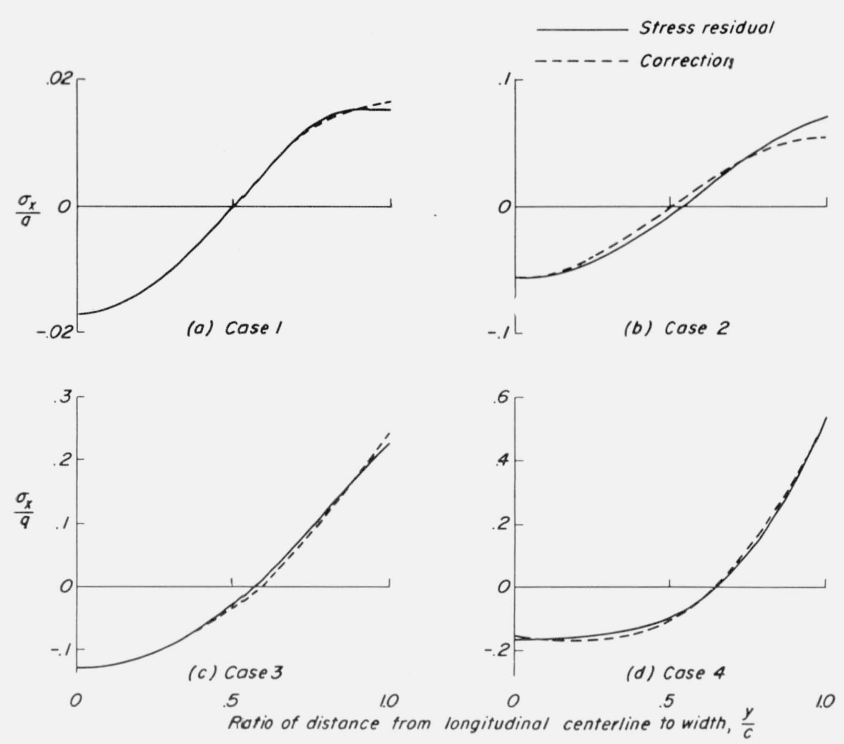

FIgURE 6. i. Stress residuals on transverse boundary of plates fo ${ }^{r}$ cases 11 to 4 .

(a) ¿Case 1; (b) Case 2; (c) Case 3; (d) Case 4.

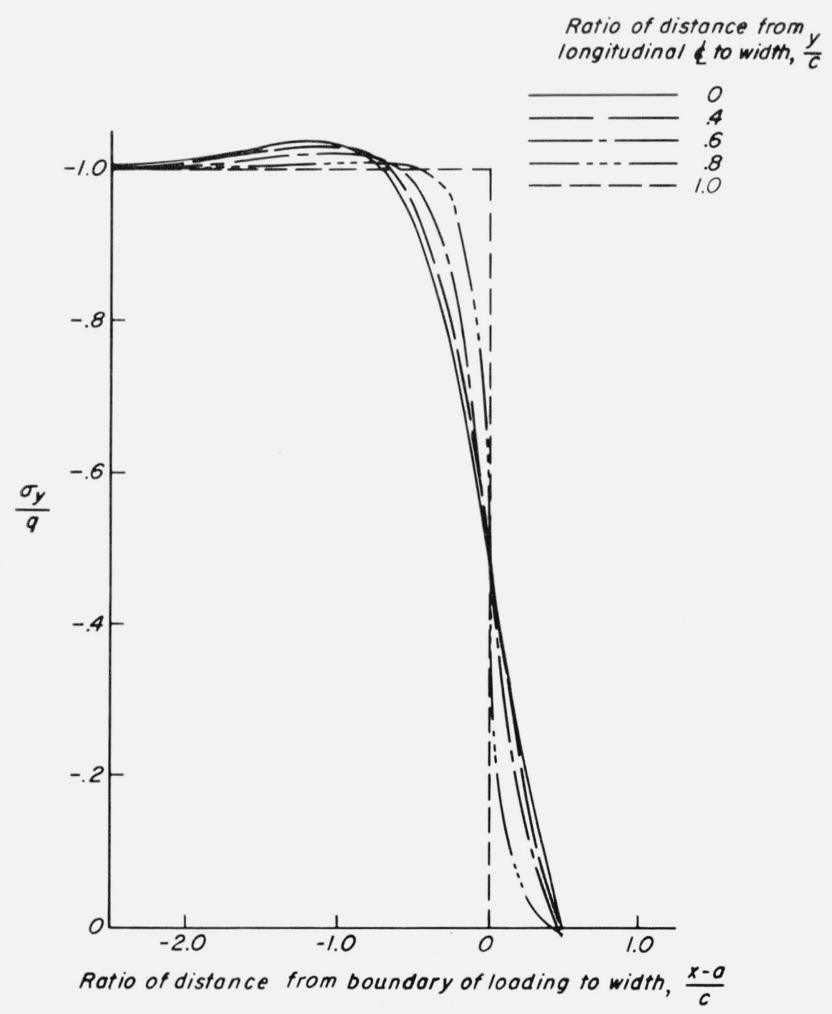

Figure 7a. Distribution of $\sigma_{\mathbf{y}} / \mathrm{q}$ in plate for case 4 .

$$
\begin{aligned}
\tau_{x y} / q & =-8 \frac{x y}{a^{2}}\left(\frac{x^{2}}{a^{2}}-1\right)\left(\frac{y^{2}}{c^{2}}-1\right)\{0.05972 \\
& \left.+0.04675\left(3 \frac{x^{2}}{a^{2}}-1\right)+0.00683\left(3 \frac{y^{2}}{a^{2}}-\frac{c^{2}}{a^{2}}\right)\right\} .
\end{aligned}
$$

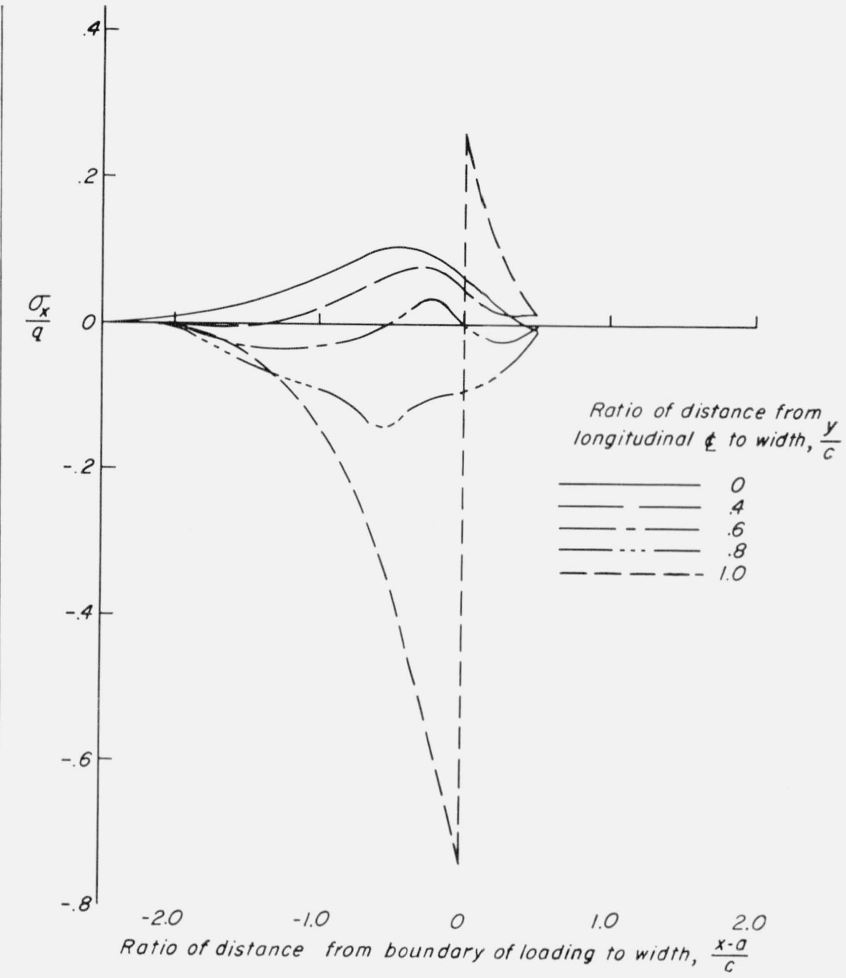

Figure 7b. Distribution of $\sigma_{\mathrm{s}} / \mathrm{q}$ in plate for case 4.

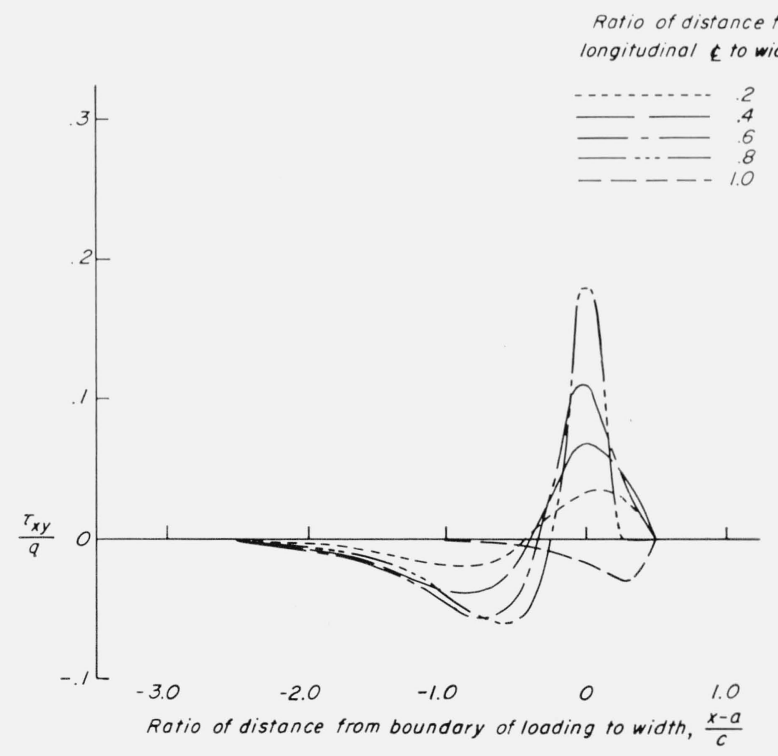

Figure 7c. Distribution of $\tau_{\mathbf{x y}} / \mathrm{q}$ in plate for case 4.

The last term in the equation for $\sigma_{x} / q$ is a constant term corresponding to a uniform load.

The equation for the distribution of stresses in the $x$-direction at the boundary of the plate $x= \pm l$ for case 4 , figure $4 \mathrm{~d}$, is

$$
\sigma_{x} /\left.q\right|_{x= \pm l}=0.77\left(1-\frac{y^{2}}{l^{2}}\right)-0.08 \cos \frac{3 \pi y}{20}-0.53048,
$$




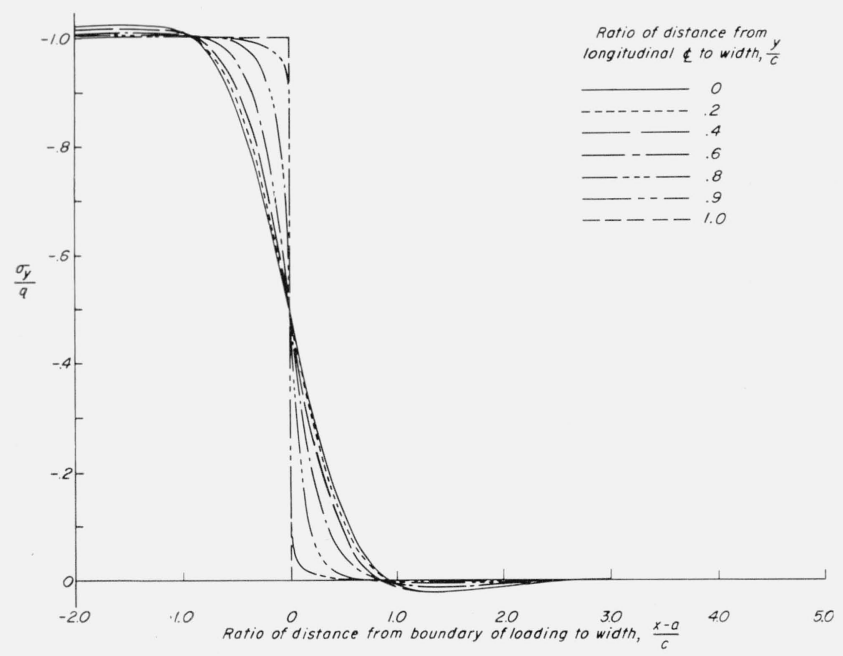

Figure 8a. Distribution of $\sigma_{\mathbf{y}} / \mathrm{q}$ in plate for case 5.

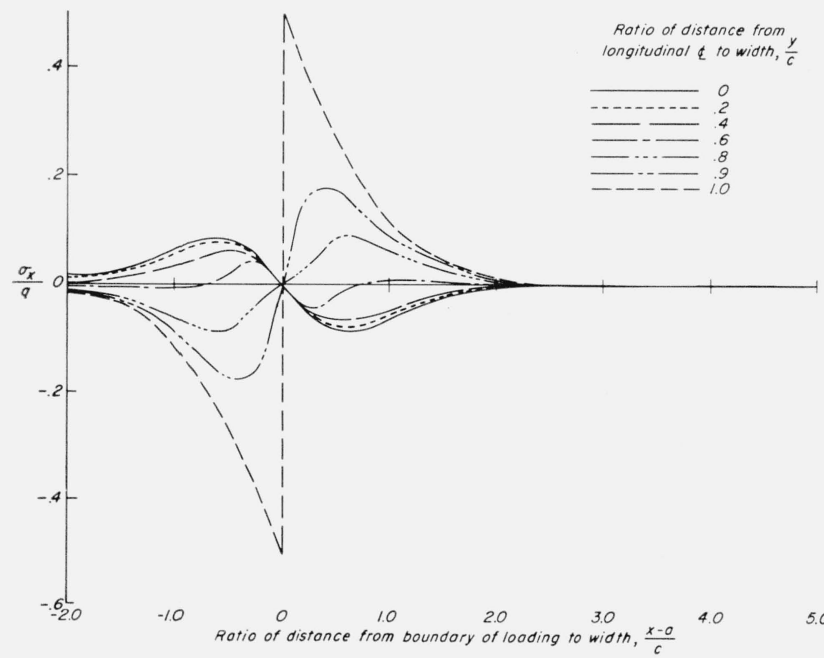

Figure 8 b. Distribution of $\sigma_{\mathbf{x}} / \mathrm{q}$ in plate for case 5 .

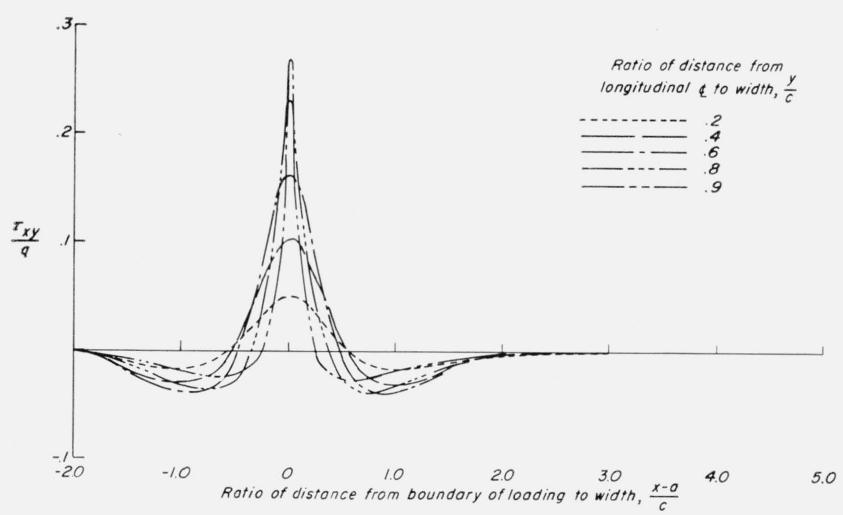

Figure 8c. Distribution of $\tau_{\mathbf{x y}} / \mathrm{q}$ in plate for case 5.

which is a combination of a parabolic distribution and a cosine distribution. The corresponding stresses in other regions of the plate were obtained by superposing the stresses due to the cosine dis-

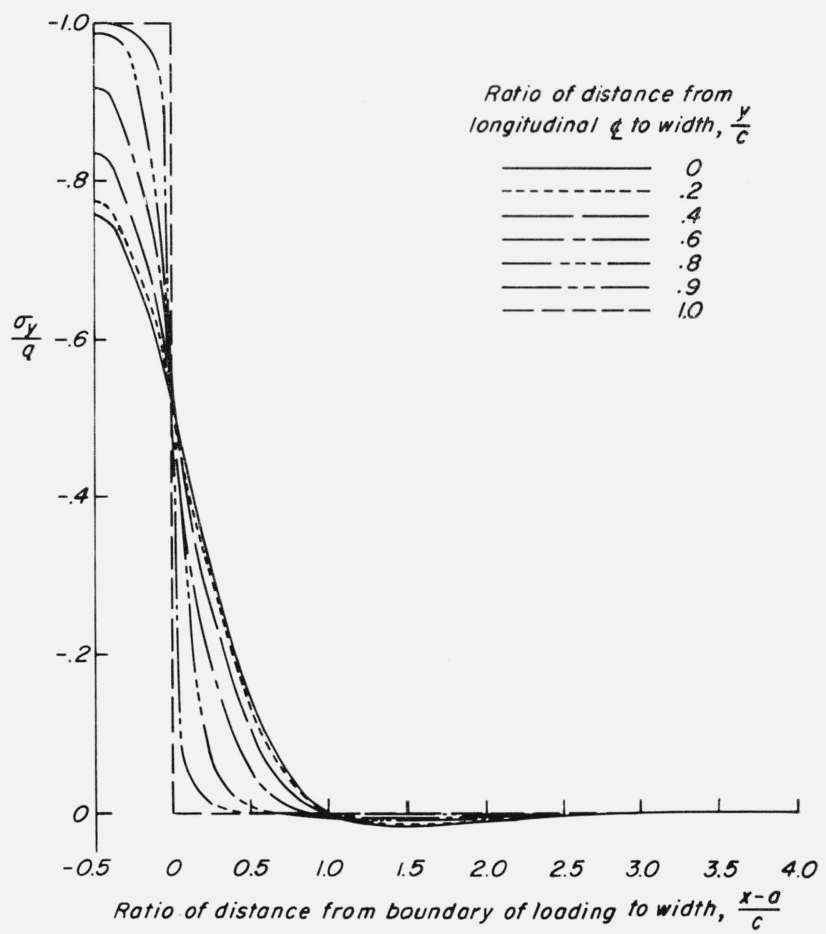

Figure 9a. Distribution of $\sigma_{\mathbf{y}} / \mathrm{q}$ in plate for case 8.

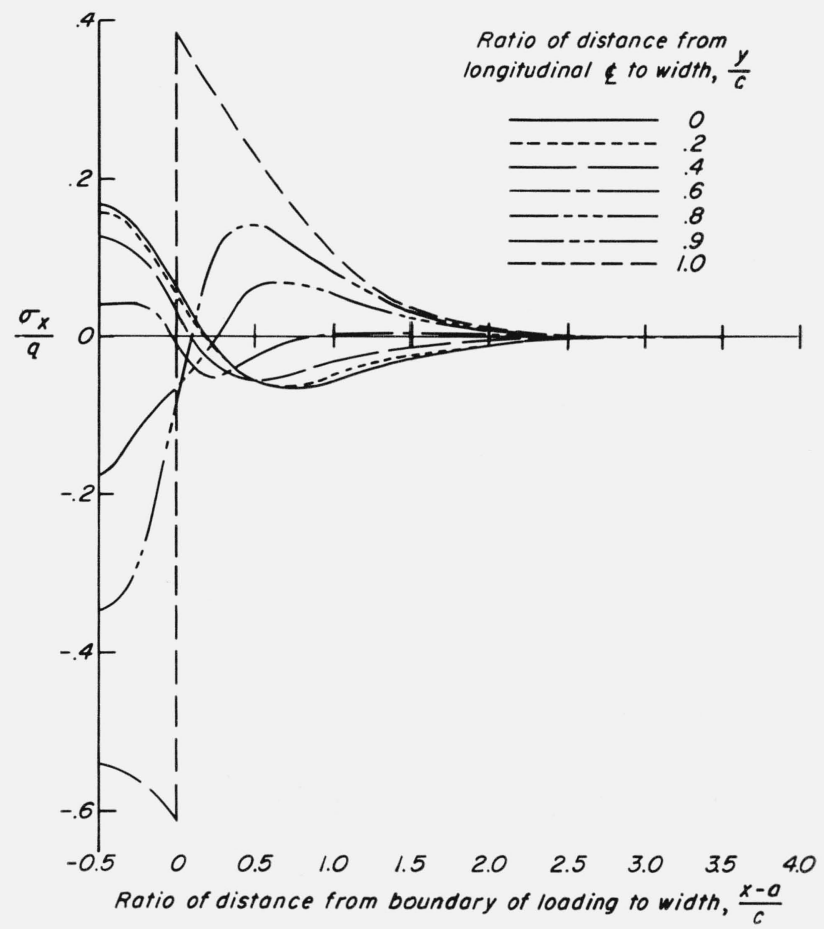

Figure 9b. Distribution of $\sigma_{\mathbf{x}} / \mathrm{q}$ in plate for case 8.

tribution on those corresponding to the parabolic distribution as obtained from solutions similar to those for cases 1 and 2 and case 3, respectively. These stresses and those corresponding to a uniform load, $\sigma_{x} / q=-0.53048$, were added to the computed stresses to give essentially zero stress on the boundary at $x= \pm l$. 


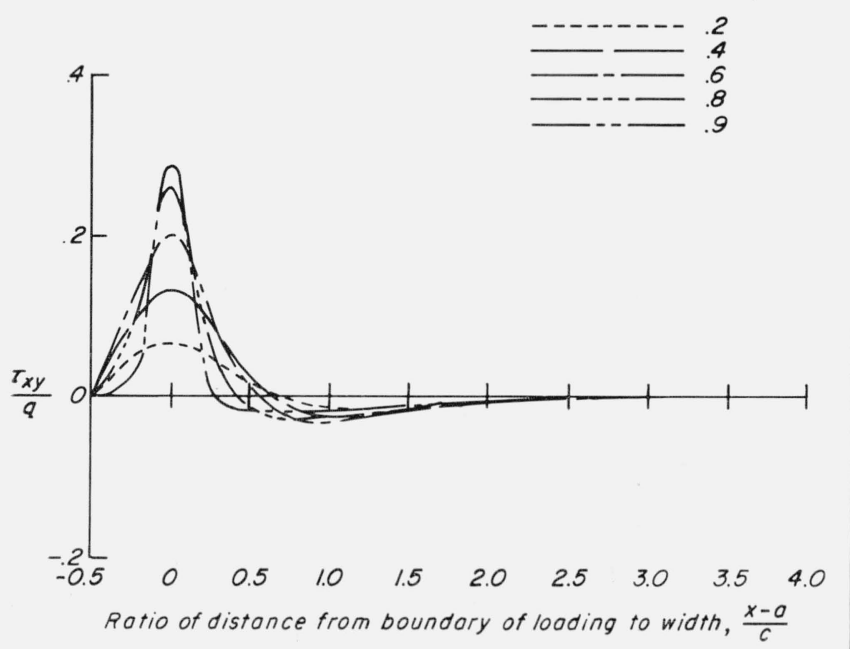

Figure 9c. Distribution of $\tau_{\mathbf{x y}} / \mathrm{q}$ in plate for case 8.

\section{Results}

Values of the ratios of stress to applied load per unit area, $\sigma_{x} / q, \sigma_{y} / q$, and $\tau_{x y} / q$, were computed so that the results would be applicable for any value of load q. In the remainder of the paper, these ratios will be referred to as stresses.

Typical plots of the stresses $\frac{\sigma_{x}}{q}, \frac{\sigma_{y}}{q}$, and $\frac{\tau_{x y}}{q}$ as corrected are shown in figures 7,8 , and 9 . The maximum and minimum stresses are listed in table 1.

The computed values of stresses are given in tables 2 to 9 . These tables are presented in terms of nondimensional parameters for generality. Since the stress distribution in the plate is affected only in the region of load discontinuity, one of the parameters used is $\frac{x-a}{c}$ where $(x-a)$ is the distance measured from the point of discontinuity of the load $p$ in figure $4 \mathrm{c}$ or figure 5 .
TABLE 2. Stresses in plate for case 1

$\frac{\text { Unloaded length }}{\text { Width }}, \frac{l-a}{c}=2.0 ; l>>c$

\begin{tabular}{|c|c|c|c|c|c|c|c|c|}
\hline \multirow{2}{*}{$\begin{array}{l}\text { No. of } \\
\text { terms, } \\
\quad m\end{array}$} & \multirow{2}{*}{$\frac{x-a}{c}$} & \multirow{2}{*}{$y$} & \multicolumn{3}{|c|}{704 Results } & \multicolumn{3}{|c|}{ Corrected } \\
\hline & & & $\sigma_{x} / q$ & $\sigma_{y} / q$ & $\tau_{x y} / q$ & $\sigma_{x} / q$ & $\sigma_{y} / q$ & $\tau_{x y} / q$ \\
\hline $\begin{array}{r}35 \\
44 \\
59 \\
89 \\
100 \\
19\end{array}$ & -2.5 & $\begin{array}{r}0 \\
0.2 \\
.4 \\
.6 \\
.8 \\
1.0\end{array}$ & $\begin{array}{c}0.002 \\
.001 \\
0 \\
-0.001 \\
-.001 \\
0\end{array}$ & $\begin{array}{l}-1.004 \\
-1.004 \\
-1.003 \\
-1.002 \\
-1.000 \\
-1.0\end{array}$ & $\begin{array}{c}0 \\
-0.001 \\
-.002 \\
-.002 \\
-.001 \\
0\end{array}$ & $\begin{array}{c}0.002 \\
.001 \\
0 \\
-0.001 \\
-.001 \\
0\end{array}$ & $\begin{array}{l}-1.004 \\
-1.004 \\
-1.003 \\
-1.002 \\
-1.000 \\
-1.0\end{array}$ & $\begin{array}{c}0 \\
-0.001 \\
-.002 \\
-.002 \\
-.001 \\
0\end{array}$ \\
\hline $\begin{array}{r}35 \\
44 \\
59 \\
89 \\
100 \\
19\end{array}$ & -2.0 & $\begin{array}{r}0 \\
0.2 \\
.4 \\
.6 \\
.8 \\
1.0\end{array}$ & $\begin{array}{r}.008 \\
.007 \\
.003 \\
-.003 \\
-.007 \\
-.008\end{array}$ & $\begin{array}{l}-1.012 \\
-1.011 \\
-1.008 \\
-1.005 \\
-1.001 \\
-1.0\end{array}$ & $\begin{array}{c}0 \\
-0.004 \\
-.007 \\
-.008 \\
-.005 \\
0\end{array}$ & $\begin{array}{r}.008 \\
.007 \\
.003 \\
-.003 \\
-.007 \\
-.008\end{array}$ & $\begin{array}{l}-1.012 \\
-1.011 \\
-1.008 \\
-1.005 \\
-1.001 \\
-1.0\end{array}$ & $\begin{array}{c}0 \\
-0.004 \\
-.007 \\
-.008 \\
-.005 \\
0\end{array}$ \\
\hline $\begin{array}{r}35 \\
44 \\
59 \\
89 \\
100 \\
19\end{array}$ & -1.5 & $\begin{array}{r}0 \\
0.2 \\
.4 \\
.6 \\
.8 \\
1.0\end{array}$ & $\begin{array}{r}.028 \\
.023 \\
-.011 \\
-.006 \\
-.025 \\
-.036\end{array}$ & $\begin{array}{l}-1.022 \\
-1.021 \\
-1.016 \\
-1.010 \\
-1.003 \\
-1.0\end{array}$ & $\begin{array}{c}0 \\
-0.011 \\
-.019 \\
-.021 \\
-.015\end{array}$ & $\begin{array}{r}.028 \\
.023 \\
.011 \\
-.006 \\
-.025 \\
-.036\end{array}$ & $\begin{array}{l}-1.022 \\
-1.021 \\
-1.016 \\
-1.010 \\
-1.003 \\
-1.0\end{array}$ & $\begin{array}{c}0 \\
-0.011 \\
-.019 \\
-.021 \\
-.015 \\
0\end{array}$ \\
\hline $\begin{array}{r}35 \\
44 \\
59 \\
89 \\
100 \\
19\end{array}$ & $\begin{array}{l}-1.0 \\
\end{array}$ & $\begin{array}{r}0 \\
0.2 \\
.4 \\
.6 \\
.8 \\
1.0\end{array}$ & $\begin{array}{r}0.064 \\
.057 \\
.033 \\
-.008 \\
-.061 \\
-.113\end{array}$ & $\begin{array}{l}-1.012 \\
-1.012 \\
-1.012 \\
-1.010 \\
-1.004 \\
-1.0\end{array}$ & $\begin{array}{c}0 \\
-0.016 \\
-.030 \\
-.038 \\
-.031 \\
0\end{array}$ & $\begin{array}{r}0.064 \\
.057 \\
.033 \\
-.008 \\
-.061 \\
-.013\end{array}$ & $\begin{array}{l}-1.012 \\
-1.012 \\
-1.012 \\
-1.010 \\
-1.004 \\
-1.0\end{array}$ & $\begin{array}{c}0 \\
-0.016 \\
-.030 \\
-.038 \\
-.031 \\
0\end{array}$ \\
\hline $\begin{array}{r}35 \\
44 \\
59 \\
89 \\
100 \\
19\end{array}$ & -0.75 & $\begin{array}{r}0 \\
0.2 \\
.4 \\
.6 \\
.8 \\
1.0\end{array}$ & $\begin{array}{r}.082 \\
.074 \\
.050 \\
0 \\
-0.082 \\
-.181\end{array}$ & $\begin{array}{l}-0.971 \\
-.975 \\
-.987 \\
-.999 \\
-1.003 \\
-1.0\end{array}$ & $\begin{array}{c}0 \\
-0.010 \\
-.023 \\
-.037 \\
-.037 \\
0\end{array}$ & $\begin{array}{r}.082 \\
.074 \\
.050 \\
0 \\
-0.082 \\
-.181\end{array}$ & $\begin{array}{l}-0.971 \\
-.975 \\
-.987 \\
-.999 \\
-1.003 \\
-1.0\end{array}$ & $\begin{array}{c}0 \\
-0.010 \\
-.023 \\
-.037 \\
-.037 \\
0\end{array}$ \\
\hline $\begin{array}{r}35 \\
44 \\
59 \\
89 \\
100 \\
19\end{array}$ & -0.5 & $\begin{array}{r}0 \\
0.2 \\
.4 \\
.6 \\
.8 \\
1.0\end{array}$ & $\begin{array}{r}.084 \\
.080 \\
.064 \\
.020 \\
-.088 \\
-.271\end{array}$ & $\begin{array}{c}-0.880 \\
-.889 \\
-.918 \\
-.960 \\
-.994 \\
-1.0\end{array}$ & $\begin{array}{l}0 \\
0.007 \\
.007 \\
-.007 \\
-.028 \\
0\end{array}$ & $\begin{array}{r}.084 \\
.080 \\
.064 \\
.020 \\
-.088 \\
-.271\end{array}$ & $\begin{array}{r}-0.880 \\
-.889 \\
-.918 \\
-.960 \\
-.994 \\
-1.0\end{array}$ & $\begin{array}{l}0 \\
0.007 \\
.007 \\
-.007 \\
-.028 \\
0\end{array}$ \\
\hline $\begin{array}{r}35 \\
44 \\
59 \\
89 \\
100 \\
19\end{array}$ & -0.25 & $\begin{array}{r}0 \\
0.2 \\
.4 \\
.6 \\
.8 \\
1.0\end{array}$ & $\begin{array}{r}0.056 \\
.056 \\
.055 \\
.043 \\
-.035 \\
-.380\end{array}$ & $\begin{array}{c}-0.718 \\
-.728 \\
-.763 \\
-.832 \\
-.944 \\
-1.0\end{array}$ & $\begin{array}{l}0 \\
0.035 \\
.066 \\
.080 \\
.042 \\
0\end{array}$ & $\begin{array}{r}0.056 \\
.056 \\
.055 \\
.043 \\
-.036 \\
-.380\end{array}$ & $\begin{array}{c}-0.718 \\
-.729 \\
-.763 \\
-.832 \\
-.944 \\
-1.0\end{array}$ & $\begin{array}{l}0 \\
0.035 \\
.065 \\
.079 \\
.041 \\
0\end{array}$ \\
\hline
\end{tabular}

TABLE 2. Stresses in plate for case 1-Continued

\begin{tabular}{|c|c|c|c|c|c|c|c|c|}
\hline \multirow{2}{*}{$\begin{array}{l}\text { No. of } \\
\text { terms, } \\
m\end{array}$} & \multirow{2}{*}{$\frac{x-a}{c}$} & \multirow{2}{*}{$\frac{y}{c}$} & \multicolumn{3}{|c|}{704 Results } & \multicolumn{3}{|c|}{ Corrected } \\
\hline & & & $\sigma_{x} / q$ & $\sigma_{y} / q$ & $\tau_{x y} / q$ & $\sigma_{x} / q$ & $\sigma_{y} / q$ & $\tau_{x y} / q$ \\
\hline $\begin{array}{r}35 \\
44 \\
59 \\
89 \\
100\end{array}$ & 0 & $\begin{array}{l}0 \\
0.2 \\
.4 \\
.6 \\
.8\end{array}$ & $\begin{array}{c}0 \\
0 \\
0 \\
0 \\
0 \\
-0.500\end{array}$ & $\begin{array}{r}-0.500 \\
-.500 \\
-.500 \\
-.500 \\
-.500 \\
-1.0\end{array}$ & $\begin{array}{l}0 \\
0.050 \\
.103 \\
.162 \\
.232\end{array}$ & $\begin{array}{c}0 \\
0 \\
0 \\
0 \\
0 \\
-0.500\end{array}$ & $\begin{array}{r}-0.500 \\
-.500 \\
-.500 \\
-.500 \\
-.500 \\
-1.0\end{array}$ & $\begin{array}{l}0 \\
0.050 \\
.103 \\
.162 \\
.232\end{array}$ \\
\hline 19 & & 1.0 & $\left\{\begin{array}{r}-0.000 \\
.500\end{array}\right.$ & $\left\{\begin{array}{c}-1.0 \\
0\end{array}\right.$ & 0 & $\left\{\begin{array}{r}-0.500 \\
.500\end{array}\right.$ & -1.0 & 0 \\
\hline $\begin{array}{r}35 \\
44 \\
59 \\
89 \\
100 \\
19\end{array}$ & 0.25 & $\begin{array}{r}0 \\
0.2 \\
.4 \\
.6 \\
.8 \\
1.0\end{array}$ & $\begin{array}{r}-.056 \\
-.056 \\
-.055 \\
-.043 \\
.035 \\
.379\end{array}$ & $\begin{array}{l}-0.282 \\
-.272 \\
-.237 \\
-.168 \\
-.056 \\
0\end{array}$ & $\begin{array}{l}0 \\
0.035 \\
.066 \\
.079 \\
.042 \\
0\end{array}$ & $\begin{array}{r}-.055 \\
-.056 \\
-.055 \\
-.044 \\
.035 \\
.379\end{array}$ & $\begin{array}{c}-0.282 \\
-.272 \\
-.237 \\
-.168 \\
-.056\end{array}$ & $\begin{array}{l}0 \\
0.035 \\
.065 \\
.079 \\
.041 \\
0\end{array}$ \\
\hline $\begin{array}{r}35 \\
44 \\
59 \\
89 \\
100 \\
19\end{array}$ & 0.5 & $\begin{array}{r}0 \\
0.2 \\
.4 \\
.6 \\
.8 \\
1.0\end{array}$ & $\begin{array}{r}-0.083 \\
-.080 \\
-.064 \\
-.020 \\
.087 \\
.270\end{array}$ & $\begin{array}{l}-0.120 \\
-.111 \\
-.082 \\
-.040 \\
-.006 \\
0\end{array}$ & $\begin{array}{c}0 \\
0.007 \\
.007 \\
-.007 \\
-.028 \\
0\end{array}$ & $\begin{array}{r}-0.083 \\
-.079 \\
-.064 \\
-.020 \\
.087 \\
.269\end{array}$ & $\begin{array}{l}-0.121 \\
-. .11 \\
-.082 \\
-.040 \\
-.005 \\
0\end{array}$ & $\begin{array}{l}0 \\
0.007 \\
.007 \\
-.008 \\
-.029 \\
0\end{array}$ \\
\hline $\begin{array}{r}35 \\
44 \\
59 \\
89 \\
100 \\
19\end{array}$ & 0.75 & $\begin{array}{r}0 \\
0.2 \\
.4 \\
.6 \\
.8 \\
1.0\end{array}$ & $\begin{array}{c}-.081 \\
-.074 \\
-.050 \\
0 \\
0.082 \\
.180\end{array}$ & $\begin{array}{c}-0.029 \\
-.024 \\
-.013 \\
-.001 \\
.003 \\
0\end{array}$ & $\begin{array}{l}0 \\
-0.010 \\
-.023 \\
-.037 \\
-.037 \\
0\end{array}$ & $\begin{array}{r}-0.080 \\
-.073 \\
-.049 \\
0 \\
0.080 \\
.179\end{array}$ & $\begin{array}{r}-0.030 \\
-.025 \\
-.013 \\
-.001 \\
.003 \\
0\end{array}$ & $\begin{array}{l}0 \\
-0.011 \\
-.024 \\
-.038 \\
-.038 \\
0\end{array}$ \\
\hline $\begin{array}{r}35 \\
44 \\
59 \\
89 \\
100 \\
19\end{array}$ & 1.0 & $\begin{array}{r}0 \\
0.2 \\
.4 \\
.6 \\
.8 \\
1.0\end{array}$ & $\begin{array}{r}-.064 \\
-.056 \\
-.033 \\
.008 \\
.061 \\
.112\end{array}$ & $\begin{array}{l}0.012 \\
.013 \\
.013 \\
.010 \\
.004 \\
0\end{array}$ & $\begin{array}{c}0 \\
-0.016 \\
-.030 \\
-.038 \\
-.031 \\
0\end{array}$ & $\begin{array}{r}-.061 \\
-.054 \\
-.032 \\
.007 \\
.058 \\
.09\end{array}$ & $\begin{array}{l}0.011 \\
.011 \\
.012 \\
.011 \\
.006 \\
0\end{array}$ & $\begin{array}{c}0 \\
-0.017 \\
-.032 \\
-.040 \\
-.033 \\
0\end{array}$ \\
\hline $\begin{array}{r}35 \\
44 \\
59 \\
89 \\
100 \\
19\end{array}$ & 1.5 & $\begin{array}{r}0 \\
0.2 \\
.4 \\
.6 \\
.8 \\
1.0\end{array}$ & $\begin{array}{r}-0.029 \\
-.025 \\
-.011 \\
.007 \\
.026 \\
.036\end{array}$ & $\begin{array}{l}0.026 \\
.024 \\
.019 \\
.011 \\
.004 \\
0\end{array}$ & $\begin{array}{l}0 \\
-0.010 \\
-.017 \\
-.019 \\
-.014 \\
0\end{array}$ & $\begin{array}{r}-0.020 \\
-.017 \\
-.009 \\
.004 \\
.018 \\
.027\end{array}$ & $\begin{array}{r}0.024 \\
.023 \\
.018 \\
.012 \\
.005 \\
0\end{array}$ & $\begin{array}{l}0 \\
-0.013 \\
-.022 \\
-.024 \\
-.017 \\
0\end{array}$ \\
\hline $\begin{array}{r}35 \\
44 \\
59 \\
89 \\
100 \\
19\end{array}$ & 2.0 & $\begin{array}{r}0 \\
0.2 \\
.4 \\
.6 \\
.8 \\
1.0\end{array}$ & $\begin{array}{r}-.017 \\
-.014 \\
-.005 \\
.005 \\
.014 \\
.015\end{array}$ & $\begin{array}{l}.023 \\
.021 \\
.016 \\
.009 \\
.003 \\
0 .\end{array}$ & $\begin{array}{l}0 \\
0 \\
0 \\
0 \\
0 \\
0\end{array}$ & $\begin{array}{l}0 \\
0 \\
0 \\
0 \\
0 \\
0\end{array}$ & $\begin{array}{r}0.040 \\
.035 \\
.021 \\
.004 \\
-.011 \\
-.017\end{array}$ & $\begin{array}{l}0 \\
0 \\
0 \\
0 \\
0 \\
0\end{array}$ \\
\hline
\end{tabular}


Table 3. Stresses in plate for case 2

$\frac{\text { Unloaded length }}{\text { Width }}, \frac{l-a}{c}=1.5 ; l>c$

\begin{tabular}{|c|c|c|c|c|c|c|c|c|}
\hline \multirow{2}{*}{$\begin{array}{l}\text { No. of } \\
\text { terms, } \\
m\end{array}$} & \multirow{2}{*}{$\frac{x-a}{c}$} & \multirow{2}{*}{$\frac{y}{c}$} & \multicolumn{3}{|c|}{704 Results } & \multicolumn{3}{|c|}{ Corrected } \\
\hline & & & $\sigma_{x} / q$ & $\sigma_{y} / q$ & $\tau_{x y} / q$ & $\sigma_{x} / q$ & $\sigma_{y} / q$ & $\tau_{x y} / q$ \\
\hline $\begin{array}{r}33 \\
41 \\
55 \\
82 \\
100 \\
18\end{array}$ & \begin{tabular}{c}
-2.5 \\
\hdashline-2 \\
-
\end{tabular} & $\begin{array}{r}0 \\
0.2 \\
.4 \\
.6 \\
.8 \\
1.0\end{array}$ & $\begin{array}{c}0.002 \\
0 \\
-001 \\
-0.001 \\
-.001 \\
0\end{array}$ & $\begin{array}{l}-1.004 \\
-1.004 \\
-1.003 \\
-1.002 \\
-1.000 \\
-1.0\end{array}$ & $\begin{array}{c}0 \\
-0.001 \\
-.002 \\
-.002 \\
-.001 \\
0\end{array}$ & $\begin{array}{c}0.002 \\
.001 \\
0 \\
-0.001 \\
-.001 \\
0\end{array}$ & $\begin{array}{l}-1.004 \\
-1.004 \\
-1.003 \\
-1.002 \\
-1.000 \\
-1.0\end{array}$ & $\begin{array}{l}0 \\
-0.001 \\
-.002 \\
-.002 \\
-.001 \\
0\end{array}$ \\
\hline $\begin{array}{r}33 \\
41 \\
55 \\
82 \\
100 \\
18\end{array}$ & -2.0 & $\begin{array}{r}0 \\
0.2 \\
.4 \\
.6 \\
.8 \\
1.0\end{array}$ & $\begin{array}{r}0.008 \\
.007 \\
.003 \\
-.003 \\
-.007 \\
-.008\end{array}$ & $\begin{array}{l}-1.012 \\
-1.011 \\
-1.008 \\
-1.005 \\
-1.001 \\
-1.0\end{array}$ & $\begin{array}{c}0 \\
-0.004 \\
-.007 \\
-.008 \\
-.005 \\
0\end{array}$ & $\begin{array}{r}0.008 \\
.007 \\
.003 \\
-.003 \\
-.007 \\
-.008\end{array}$ & $\begin{array}{l}-1.012 \\
-1.011 \\
-1.008 \\
-1.005 \\
-1.001 \\
-1.0\end{array}$ & $\begin{array}{c}0 \\
-0.004 \\
-.007 \\
-.008 \\
-.005 \\
0\end{array}$ \\
\hline $\begin{array}{r}33 \\
41 \\
55 \\
82 \\
100 \\
18\end{array}$ & -1.5 & $\begin{array}{r}0 \\
0.2 \\
.4 \\
.6 \\
.8 \\
1.0\end{array}$ & $\begin{array}{r}.028 \\
.023 \\
.011 \\
-.006 \\
-.025 \\
-.036\end{array}$ & $\begin{array}{l}-1.022 \\
-1.021 \\
-1.016 \\
-1.010 \\
-1.003 \\
-1.0\end{array}$ & $\begin{array}{c}0 \\
-0.011 \\
-.019 \\
-.021 \\
-.015 \\
0\end{array}$ & $\begin{array}{r}.028 \\
.024 \\
.011 \\
-.006 \\
-.025 \\
-.036\end{array}$ & $\begin{array}{l}-1.022 \\
-1.021 \\
-1.016 \\
-1.010 \\
-1.003 \\
-1.0\end{array}$ & $\begin{array}{c}0 \\
-0.011 \\
-.019 \\
-.021 \\
-.015 \\
0\end{array}$ \\
\hline $\begin{array}{r}33 \\
41 \\
55 \\
82 \\
100 \\
18\end{array}$ & $\begin{array}{c}-1.0 \\
\end{array}$ & $\begin{array}{r}0 \\
0.2 \\
.4 \\
.6 \\
.8 \\
1.0\end{array}$ & $\begin{array}{r}0.064 \\
.057 \\
.033 \\
-.008 \\
-.061 \\
-.0114\end{array}$ & $\begin{array}{l}-1.011 \\
-1.012 \\
-1.012 \\
-1.010 \\
-1.004 \\
-1.0\end{array}$ & $\begin{array}{c}0 \\
-0.016 \\
-.030 \\
-.038 \\
-.031 \\
0\end{array}$ & $\begin{array}{r}0.065 \\
.057 \\
.033 \\
-.008 \\
-.061 \\
-.0114\end{array}$ & $\begin{array}{l}-1.011 \\
-1.012 \\
-1.012 \\
-1.010 \\
-1.004 \\
-1.0\end{array}$ & $\begin{array}{c}0 \\
-0.016 \\
-.030 \\
-.038 \\
-.031 \\
0\end{array}$ \\
\hline $\begin{array}{r}33 \\
41 \\
55 \\
82 \\
100 \\
18\end{array}$ & \begin{tabular}{c}
-0.75 \\
\hdashline- \\
\hdashline-
\end{tabular} & $\begin{array}{r}0 \\
0.2 \\
.4 \\
.6 \\
.8 \\
1.0\end{array}$ & $\begin{array}{r}.082 \\
.075 \\
.050 \\
0 \\
-0.082 \\
-.181\end{array}$ & $\begin{array}{l}-0.971 \\
-.975 \\
-.987 \\
-.999 \\
-1.003 \\
-1.0\end{array}$ & $\begin{array}{c}0 \\
-0.010 \\
-.023 \\
-.037 \\
-.037 \\
0\end{array}$ & $\begin{array}{r}.082 \\
.075 \\
.050 \\
0 \\
-0.083 \\
-.182\end{array}$ & $\begin{array}{c}-0.971 \\
-.976 \\
-.987 \\
-.999 \\
-1.002 \\
-1.0\end{array}$ & $\begin{array}{c}0 \\
-0.011 \\
-.024 \\
-.037 \\
-.037 \\
0\end{array}$ \\
\hline $\begin{array}{r}33 \\
41 \\
55 \\
82 \\
100 \\
18\end{array}$ & \begin{tabular}{c}
-0.5 \\
\hdashline-0 \\
\end{tabular} & $\begin{array}{r}0 \\
0.2 \\
.4 \\
.6 \\
.8 \\
1.0\end{array}$ & $\begin{array}{r}.084 \\
.080 \\
.065 \\
.020 \\
-.088 \\
-.271\end{array}$ & $\begin{array}{r}-0.879 \\
-.889 \\
-.918 \\
-.960 \\
-.994 \\
-1.0\end{array}$ & $\begin{array}{l}0 \\
0.007 \\
.007 \\
-.007 \\
-.028 \\
0\end{array}$ & $\begin{array}{r}.085 \\
.081 \\
.065 \\
.020 \\
-.088 \\
-.272\end{array}$ & $\begin{array}{c}-0.880 \\
-.890 \\
-.918 \\
-.960 \\
-.994 \\
-1.0\end{array}$ & $\begin{array}{r}0 \\
0.007 \\
-.007 \\
-.008 \\
-.029 \\
0\end{array}$ \\
\hline $\begin{array}{r}33 \\
41 \\
55 \\
82 \\
100 \\
18\end{array}$ & \begin{tabular}{c}
-0.25 \\
\hdashline-1 \\
\hdashline-1
\end{tabular} & $\begin{array}{r}0 \\
0.2 \\
.4 \\
.6 \\
.8 \\
1.0\end{array}$ & $\begin{array}{r}0.056 \\
.056 \\
.056 \\
.043 \\
-.036 \\
-.380\end{array}$ & $\begin{array}{c}-0.718 \\
-.728 \\
-.762 \\
-.832 \\
-.944 \\
-1.0\end{array}$ & $\begin{array}{l}0 \\
0.035 \\
.066 \\
.080 \\
.042 \\
0\end{array}$ & $\begin{array}{r}0.058 \\
.058 \\
.056 \\
.043 \\
-.037 \\
-.382\end{array}$ & $\begin{array}{c}-0.719 \\
-.729 \\
-.763 \\
-.832 \\
-.943 \\
-1.0\end{array}$ & $\begin{array}{l}0 \\
0.034 \\
.065 \\
.078 \\
.041 \\
0\end{array}$ \\
\hline $\begin{array}{r}33 \\
41 \\
55 \\
82 \\
100 \\
18\end{array}$ & - & $\begin{array}{r}0 \\
0.2 \\
.4 \\
.6 \\
.8 \\
1.0\end{array}$ & $\begin{array}{c}0 \\
0 \\
0 \\
0 \\
0 \\
\left\{\begin{array}{r}-0.501 \\
.499\end{array}\right.\end{array}$ & $\begin{array}{c}-0.499 \\
-.499 \\
-.499 \\
-.500 \\
-.500 \\
\left\{\begin{array}{c}-1.0 \\
0\end{array}\right.\end{array}$ & $\begin{array}{l}0 \\
0.050 \\
.104 \\
.163 \\
.232 \\
0\end{array}$ & $\begin{array}{r}.003 \\
.002 \\
.001 \\
-.001 \\
-.002 \\
\left\{\begin{array}{r}-.504 \\
.496\end{array}\right.\end{array}$ & $\begin{array}{c}-0.501 \\
-.501 \\
-.500 \\
-.499 \\
-.498 \\
\left\{\begin{array}{c}-1.0 \\
0\end{array}\right.\end{array}$ & $\begin{array}{l}0 \\
0.049 \\
.101 \\
.160 \\
.231 \\
0^{221}\end{array}$ \\
\hline $\begin{array}{r}33 \\
41 \\
55 \\
82 \\
100 \\
18\end{array}$ & \begin{tabular}{r}
0.25 \\
\hdashline- \\
-
\end{tabular} & $\begin{array}{r}0 \\
0.2 \\
.4 \\
.6 \\
.8 \\
1.0\end{array}$ & $\begin{array}{r}-.056 \\
-.056 \\
-.055 \\
-.043 \\
.036 \\
.379\end{array}$ & $\begin{array}{r}-0.280 \\
-.270 \\
-.236 \\
-.167 \\
-.056 \\
0\end{array}$ & $\begin{array}{l}0 \\
0.036 \\
.067 \\
.080 \\
.042 \\
0 .\end{array}$ & $\begin{array}{r}-.051 \\
-.052 \\
-.054 \\
-.045 \\
.031 \\
.374\end{array}$ & $\begin{array}{r}-0.283 \\
-.272 \\
-.237 \\
-.166 \\
-.054 \\
0\end{array}$ & $\begin{array}{l}0 \\
0.033 \\
.062 \\
.076 \\
.040 \\
0\end{array}$ \\
\hline $\begin{array}{r}33 \\
41 \\
55 \\
82 \\
100 \\
18\end{array}$ & \begin{tabular}{c}
0.5 \\
\hdashline \\
-
\end{tabular} & $\begin{array}{r}0 \\
0.2 \\
.4 \\
.6 \\
.8 \\
1.0\end{array}$ & $\begin{array}{r}-0.085 \\
-.081 \\
-.065 \\
-.019 \\
.089 \\
.271\end{array}$ & $\begin{array}{l}-.116 \\
-.107 \\
-.079 \\
-.038 \\
-.005 \\
0\end{array}$ & $\begin{array}{l}0 \\
0.009 \\
.009 \\
-.005 \\
-.027 \\
0\end{array}$ & $\begin{array}{r}-0.075 \\
-.073 \\
-.062 \\
-.022 \\
.081 \\
.261\end{array}$ & $\begin{array}{r}-0.121 \\
-.111 \\
-.081 \\
-.037 \\
-.001 \\
.005\end{array}$ & $\begin{array}{l}0 \\
0.004 \\
.002 \\
-.012 \\
-.032 \\
0\end{array}$ \\
\hline $\begin{array}{r}33 \\
41 \\
55 \\
82 \\
100 \\
18\end{array}$ & \begin{tabular}{r}
0.75 \\
\hdashline-1. \\
-
\end{tabular} & $\begin{array}{r}0 \\
0.2 \\
.4 \\
.6 \\
.8 \\
1.0\end{array}$ & $\begin{array}{r}-.086 \\
-.078 \\
-.051 \\
.002 \\
.085 \\
.183\end{array}$ & $\begin{array}{r}-0.022 \\
-.018 \\
-.008 \\
.002 \\
.003 \\
0\end{array}$ & $\begin{array}{c}0 \\
-0.008 \\
-.019 \\
-.032 \\
-.034 \\
0\end{array}$ & $\begin{array}{r}-.068 \\
-.063 \\
-.045 \\
-.004 \\
.071 \\
.166\end{array}$ & $\begin{array}{r}-.029 \\
-.024 \\
-.010 \\
.004 \\
.009 \\
.007\end{array}$ & $\begin{array}{l}0 \\
-0.015 \\
-.031 \\
-.044 \\
-.042 \\
0\end{array}$ \\
\hline $\begin{array}{r}33 \\
41 \\
55 \\
82 \\
100 \\
18\end{array}$ & - & $\begin{array}{r}0 \\
0.2 \\
.4 \\
.6 \\
.8 \\
1.0\end{array}$ & $\begin{array}{r}-.073 \\
-.063 \\
-.036 \\
.010 \\
.068 \\
.121\end{array}$ & $\begin{array}{l}0.023 \\
.022 \\
.020 \\
.015 \\
.006 \\
0\end{array}$ & $\begin{array}{c}0 \\
-0.012 \\
-.023 \\
-.030 \\
-.026 \\
0\end{array}$ & $\begin{array}{r}-.043 \\
-.040 \\
-.027 \\
.001 \\
.044 \\
.092\end{array}$ & $\begin{array}{l}.016 \\
.017 \\
.018 \\
.017 \\
.011 \\
.007\end{array}$ & $\begin{array}{c}0 \\
-0.022 \\
-.040 \\
-.047 \\
-.037 \\
0\end{array}$ \\
\hline $\begin{array}{r}33 \\
41 \\
55 \\
82 \\
100 \\
18\end{array}$ & '- & $\begin{array}{r}0 \\
0.2 \\
.4 \\
.6 \\
.8 \\
1.0\end{array}$ & $\begin{array}{r}-0.056 \\
-.047 \\
-.022 \\
.013 \\
.049 \\
.072\end{array}$ & $\begin{array}{l}0.044 \\
.041 \\
.032 \\
.019 \\
.006 \\
0\end{array}$ & $\begin{array}{l}0 \\
0 \\
0 \\
0 \\
0 \\
0\end{array}$ & $\begin{array}{r}-0.001 \\
-.002 \\
-.005 \\
-.004 \\
.005 \\
.017\end{array}$ & $\begin{array}{r}0.099 \\
.086 \\
.049 \\
.002 \\
-.038 \\
-.055\end{array}$ & $\begin{array}{l}0 \\
0 \\
0 \\
0 \\
0 \\
0\end{array}$ \\
\hline
\end{tabular}

TABLE 4. Stresses in plate for case 3

Unloaded length,$\frac{l-a}{c}=1.0 ; l \gg c$

\begin{tabular}{|c|c|c|c|c|c|c|c|c|}
\hline \multirow{2}{*}{$\begin{array}{l}\text { No. of } \\
\text { terms, } \\
\quad m\end{array}$} & \multirow{2}{*}{$\frac{x-a}{c}$} & \multirow{2}{*}{$\frac{y}{c}$} & \multicolumn{3}{|c|}{704 Results } & \multicolumn{3}{|c|}{ Corrected } \\
\hline & & & $\sigma_{x} / q$ & $\sigma_{y} / q$ & $\tau_{x y} / q$ & $\sigma_{x} / q$ & $\sigma_{y} / q$ & $\tau_{x y} / q$ \\
\hline $\begin{array}{r}30 \\
38 \\
50 \\
76 \\
100 \\
100 \\
100 \\
17\end{array}$ & \begin{tabular}{|l}
-2.5 \\
\hdashline-2 \\
-
\end{tabular} & $\begin{array}{r}0 \\
0.2 \\
.4 \\
.6 \\
.7 \\
.8 \\
.9 \\
1.0\end{array}$ & $\begin{array}{c}0.002 \\
.001 \\
-0.001 \\
-.001 \\
-.001 \\
-.001 \\
0\end{array}$ & $\begin{array}{l}-1.004 \\
-1.004 \\
-1.003 \\
-1.002 \\
-1.001 \\
-1.000 \\
-1.000 \\
-1.0\end{array}$ & $\begin{array}{c}0 \\
-0.001 \\
-.002 \\
-.002 \\
-.002 \\
-.001 \\
-.001 \\
0\end{array}$ & & & \\
\hline $\begin{array}{r}30 \\
38 \\
50 \\
76 \\
100 \\
100 \\
100 \\
17\end{array}$ & \begin{tabular}{c}
-2.0 \\
\hdashline-10 \\
-
\end{tabular} & $\begin{array}{r}0 \\
0.2 \\
.4 \\
.6 \\
.7 \\
.8 \\
.9 \\
1.0\end{array}$ & $\begin{array}{r}0.009 \\
.007 \\
.003 \\
-.003 \\
-.005 \\
-.007 \\
-.008 \\
-.008\end{array}$ & $\begin{array}{l}-1.012 \\
-1.011 \\
-1.008 \\
-1.005 \\
-1.003 \\
-1.001 \\
-1.000 \\
-1.0\end{array}$ & $\begin{array}{c}0 \\
-0.004 \\
-.007 \\
-.008 \\
-.007 \\
-.005 \\
-.003 \\
0\end{array}$ & & & \\
\hline $\begin{array}{r}30 \\
38 \\
50 \\
76 \\
100 \\
100 \\
100 \\
17\end{array}$ & \begin{tabular}{l}
-1.5 \\
\hdashline-1 \\
\\
\hdashline
\end{tabular} & $\begin{array}{r}0 \\
0.2 \\
.4 \\
.6 \\
.7 \\
.8 \\
.9 \\
1.0\end{array}$ & $\begin{array}{r}0.028 \\
.024 \\
.011 \\
-.006 \\
-.016 \\
-.025 \\
-.032 \\
-.036\end{array}$ & $\begin{array}{l}-1.022 \\
-1.021 \\
-1.016 \\
-1.010 \\
-1.006 \\
-1.003 \\
-1.001 \\
-1.0\end{array}$ & $\begin{array}{c}0 \\
-0.011 \\
-.019 \\
-.021 \\
-.019 \\
-.015 \\
-.009 \\
0\end{array}$ & & & \\
\hline $\begin{array}{r}30 \\
38 \\
50 \\
76 \\
100 \\
100 \\
100 \\
17\end{array}$ & -1.0 & $\begin{array}{r}0 \\
0.2 \\
.4 \\
.6 \\
.7 \\
.8 \\
.9 \\
1.0\end{array}$ & $\begin{array}{r}.064 \\
.057 \\
.033 \\
-.007 \\
-.033 \\
-.061 \\
-.089 \\
-.114\end{array}$ & $\begin{array}{l}-1.010 \\
-1.011 \\
-1.011 \\
-1.010 \\
-1.007 \\
-1.004 \\
-1.002 \\
-1.0\end{array}$ & $\begin{array}{c}0 \\
-0.016 \\
-.030 \\
-.038 \\
-.037 \\
-.031 \\
-.019 \\
0\end{array}$ & $\begin{array}{r}0.077 \\
.067 \\
.038 \\
-.009 \\
-.036 \\
-.064 \\
-.089 \\
-.0107\end{array}$ & $\begin{array}{l}-1.017 \\
-1.017 \\
-1.016 \\
-1.013 \\
-1.009 \\
-1.005 \\
-1.002 \\
-1.0\end{array}$ & $\begin{array}{c}0 \\
-0.016 \\
-.030 \\
-.038 \\
-.037 \\
-.031 \\
-.019 \\
0\end{array}$ \\
\hline $\begin{array}{r}30 \\
38 \\
50 \\
76 \\
100 \\
100 \\
100 \\
17\end{array}$ & \begin{tabular}{c}
-0.75 \\
\\
\hdashline
\end{tabular} & $\begin{array}{r}0 \\
0.2 \\
.4 \\
.6 \\
.7 \\
.8 \\
.9 \\
1.0\end{array}$ & $\begin{array}{r}0.081 \\
.074 \\
.050 \\
0 \\
-0.037 \\
-.082 \\
-.132 \\
-.182\end{array}$ & $\begin{array}{r}-0.969 \\
-.973 \\
-.986 \\
-.998 \\
-1.002 \\
-1.002 \\
-1.001 \\
-1.0\end{array}$ & $\begin{array}{c}0 \\
-0.010 \\
-.022 \\
-.036 \\
-.039 \\
-.037 \\
-.024 \\
0\end{array}$ & $\begin{array}{r}0.094 \\
.085 \\
.055 \\
-.001 \\
-.040 \\
-.085 \\
-.133 \\
-.177\end{array}$ & $\begin{array}{r}-0.978 \\
-.982 \\
-.992 \\
-1.002 \\
-1.004 \\
-1.004 \\
-1.002 \\
-1.0\end{array}$ & $\begin{array}{c}0 \\
-0.011 \\
-.025 \\
-.039 \\
-.042 \\
-.039 \\
-.026 \\
0\end{array}$ \\
\hline $\begin{array}{r}30 \\
38 \\
50 \\
76 \\
100 \\
100 \\
100 \\
17\end{array}$ & \begin{tabular}{c}
-0.5 \\
\hdashline \\
\hdashline
\end{tabular} & $\begin{array}{r}0 \\
0.2 \\
.4 \\
.6 \\
.7 \\
.8 \\
.9 \\
1.0\end{array}$ & $\begin{array}{r}.082 \\
.079 \\
.064 \\
.021 \\
.022 \\
-.086 \\
-.172 \\
-.271\end{array}$ & $\begin{array}{c}-0.875 \\
-.886 \\
-.915 \\
-.959 \\
-.979 \\
-.994 \\
-1.000 \\
-1.0\end{array}$ & $\begin{array}{l}0 \\
0.009 \\
.009 \\
-.005 \\
-.017 \\
-.027 \\
-.025 \\
0\end{array}$ & $\begin{array}{r}.098 \\
.092 \\
.071 \\
.020 \\
.018 \\
-.093 \\
-.179 \\
-.274\end{array}$ & $\begin{array}{l}-0.892 \\
-.901 \\
-.928 \\
-.966 \\
-.984 \\
-.996 \\
-1.000 \\
-1.0\end{array}$ & $\begin{array}{l}0 \\
0.005 \\
.003 \\
-.013 \\
-.025 \\
-.033 \\
-.029 \\
0\end{array}$ \\
\hline $\begin{array}{r}30 \\
38 \\
50 \\
76 \\
100 \\
100 \\
100 \\
17\end{array}$ & -0.25 & $\begin{array}{r}0 \\
0.2 \\
.4 \\
.6 \\
.7 \\
.8 \\
.9 \\
1.0\end{array}$ & $\begin{array}{r}0.052 \\
.053 \\
.054 \\
.045 \\
.023 \\
-.032 \\
-.160 \\
-.377\end{array}$ & $\begin{array}{c}-0.711 \\
-.722 \\
-.758 \\
-.830 \\
-.883 \\
-.943 \\
-.989 \\
-1.0\end{array}$ & $\begin{array}{l}0 \\
0.038 \\
.070 \\
.084 \\
.074 \\
.044 \\
.002 \\
0\end{array}$ & $\begin{array}{r}0.075 \\
.073 \\
.066 \\
.044 \\
.016 \\
-.045 \\
-.178 \\
-.397\end{array}$ & $\begin{array}{r}-0.737 \\
-.746 \\
-.777 \\
-.841 \\
-.890 \\
-.946 \\
-.990 \\
-1.0\end{array}$ & $\begin{array}{r}0 \\
0.030 \\
.056 \\
.067 \\
.058 \\
.032 \\
-.006 \\
0\end{array}$ \\
\hline $\begin{array}{r}30 \\
38 \\
50 \\
76 \\
100 \\
100 \\
100\end{array}$ & 0 & $\begin{array}{l}0 \\
0.2 \\
.4 \\
.6 \\
.7 \\
.8 \\
.9\end{array}$ & $\begin{array}{r}-.008 \\
-.007 \\
-.003 \\
.003 \\
.005 \\
.007 \\
.008\end{array}$ & $\begin{array}{r}-0.488 \\
-.489 \\
-.492 \\
-.495 \\
-.497 \\
-.499 \\
-.500\end{array}$ & $\begin{array}{l}0 \\
0.055 \\
.111 \\
.170 \\
.202 \\
.237 \\
.274\end{array}$ & $\begin{array}{r}.028 \\
.025 \\
.016 \\
.001 \\
-.008 \\
-.019 \\
-.031\end{array}$ & $\begin{array}{r}-0.521 \\
-.519 \\
-.515 \\
-.509 \\
-.506 \\
-.503 \\
-.501\end{array}$ & $\begin{array}{l}0 \\
0.041 \\
.087 \\
.142 \\
.176 \\
.215 \\
.261\end{array}$ \\
\hline 17 & & 1. 0 & $\left\{\begin{array}{r}-.492 \\
.508\end{array}\right.$ & $\left\{\begin{array}{c}-1.0 \\
0\end{array}\right.$ & 0 & $\left\{\begin{array}{r}-.543 \\
\quad .457\end{array}\right.$ & $\left\{\begin{array}{c}-1.0 \\
0\end{array}\right.$ & 0 \\
\hline $\begin{array}{r}30 \\
38 \\
50 \\
76 \\
100 \\
100 \\
100 \\
17\end{array}$ & 0.25 & $\begin{array}{r}0 \\
0.2 \\
.4 \\
.6 \\
.7 \\
.8 \\
.9 \\
1.0\end{array}$ & $\begin{array}{r}-0.072 \\
-.069 \\
-.061 \\
-.039 \\
-.011 \\
.049 \\
.180 \\
.397\end{array}$ & $\begin{array}{r}-0.265 \\
-.256 \\
-.225 \\
-.161 \\
-.111 \\
-.054 \\
-.011 \\
.067\end{array}$ & $\begin{array}{l}0 \\
0.042 \\
.078 \\
.093 \\
.082 \\
.051 \\
.005 \\
0^{0}\end{array}$ & $\begin{array}{r}-0.014 \\
-.018 \\
-.030 \\
-.041 \\
-.034 \\
.003 \\
.110 \\
.300\end{array}$ & $\begin{array}{r}-0.292 \\
-.281 \\
-.245 \\
-.172 \\
-.118 \\
-.058 \\
-.012 \\
0\end{array}$ & $\begin{array}{r}0 \\
0.023 \\
.044 \\
.053 \\
.045 \\
.020 \\
-.013 \\
0\end{array}$ \\
\hline $\begin{array}{r}30 \\
38 \\
50 \\
76 \\
100 \\
100 \\
100 \\
17\end{array}$ & \begin{tabular}{c}
0.5 \\
\hdashline-2
\end{tabular} & $\begin{array}{r}0 \\
0.2 \\
.4 \\
.6 \\
.7 \\
.8 \\
.9 \\
1.0\end{array}$ & $\begin{array}{r}-.111 \\
-.103 \\
-.076 \\
-.014 \\
.039 \\
.112 \\
.205 \\
.307\end{array}$ & $\begin{array}{r}-.098 \\
-.090 \\
-.066 \\
-.030 \\
-.014 \\
-.003 \\
.001 \\
.010\end{array}$ & $\begin{array}{c}0 \\
0.018 \\
.026 \\
.014 \\
0 \\
-0.013 \\
-.017 \\
0\end{array}$ & $\begin{array}{r}-.025 \\
-.027 \\
-.030 \\
-.017 \\
.004 \\
.040 \\
.093 \\
.150\end{array}$ & $\begin{array}{r}-0.101 \\
-.093 \\
-.068 \\
-.031 \\
-.014 \\
-.003 \\
.001 \\
0\end{array}$ & $\begin{array}{c}0 \\
-0.004 \\
-.014 \\
-.032 \\
-.043 \\
-.049 \\
-.038 \\
0\end{array}$ \\
\hline
\end{tabular}


TABLE 4. Stresses in plate for case 3-Continued

\begin{tabular}{|c|c|c|c|c|c|c|c|c|}
\hline \multirow{2}{*}{$\begin{array}{l}\text { No. of } \\
\text { terms, } \\
\quad m\end{array}$} & \multirow{2}{*}{$\frac{x-a}{c}$} & \multirow{2}{*}{$\frac{y}{c}$} & \multicolumn{3}{|c|}{704 Results } & \multicolumn{3}{|c|}{ Corrected } \\
\hline & & & $\sigma_{x} / q$ & $\sigma_{y} / q$ & $\tau_{x y} / q$ & $\sigma_{x} / q$ & $\sigma_{y} / q$ & $\tau_{x y} / q$ \\
\hline 30 & 0.75 & 0 & -0.126 & -0.006 & 0 & -0.012 & 0.047 & 0 \\
\hline 38 & - & 0.2 & -.113 & -.003 & 0.004 & -.012 & .046 & -0.015 \\
\hline 50 & & .4 & -.070 & .005 & .003 & -.009 & .043 & -.030 \\
\hline 76 & & .6 & .008 & .010 & -.006 & .003 & .032 & -.044 \\
\hline 100 & & .7 & .062 & .010 & -.012 & .013 & .024 & -.047 \\
\hline 100 & & .8 & .123 & .007 & -.014 & .025 & 014 & -.043 \\
\hline 100 & & .9 & .187 & .003 & -.011 & .033 & .005 & -.028 \\
\hline 17 & $-\ldots$ & 1. 0 & .247 & -.051 & 0 & .031 & 0 & 0 \\
\hline 30 & 1.0 & 0 & -.129 & .022 & 0 & 0 & 0.176 & 0 \\
\hline 38 & & 0.2 & -.113 & .023 & 0 & 0 & .165 & 0 \\
\hline 50 & & .4 & -.066 & .024 & 0 & 0.003 & .133 & 0 \\
\hline 76 & & .6 & .015 & .020 & 0 & .009 & .083 & 0 \\
\hline 100 & & .7 & .067 & .015 & 0 & .012 & .055 & 0 \\
\hline 100 & & .8 & .122 & .009 & 0 & 011 & .029 & 0 \\
\hline 100 & & .9 & 177 & .003 & 0 & .003 & .009 & 0 \\
\hline 17 & & 1. 0 & .227 & .065 & 0 & -.019 & 0 & 0 \\
\hline
\end{tabular}

TABLE 5. Stresses in plate for case 4

$\frac{\text { Unloaded length }}{\text { Width }}, \frac{l-a}{c}=0.5 ; l \gg c$

\begin{tabular}{|c|c|c|c|c|c|c|c|c|}
\hline \multirow{2}{*}{$\begin{array}{l}\text { No. of } \\
\text { terms, } \\
\quad m\end{array}$} & \multirow{2}{*}{$\frac{x-a}{c}$} & \multirow{2}{*}{$\frac{y}{c}$} & \multicolumn{3}{|c|}{704 Results } & \multicolumn{3}{|c|}{ Corrected } \\
\hline & & & $\sigma_{x} / q$ & $\sigma_{y} / q$ & $\tau_{x y} / q$ & $\sigma_{x} / q$ & $\sigma_{y} / q$ & $\tau_{x y} / q$ \\
\hline 28 & -2.5 & 0 & 0.002 & -1.004 & 0 & & & \\
\hline 35 & & 0.2 & .001 & -1.004 & -0.001 & & & \\
\hline 46 & - & .4 & 0 & -1.003 & -.002 & & & \\
\hline 70 & & .6 & -0.001 & -1.002 & -.002 & & & \\
\hline 93 & $-\ldots$ & .7 & -.001 & -1.001 & -.002 & & & \\
\hline 100 & 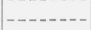 & .8 & -.001 & -1.000 & -.001 & & & \\
\hline 100 & - non & .9 & -.001 & -1.000 & -.001 & & & \\
\hline 15 & & 1.0 & -.001 & -1.0 & 0 & & & \\
\hline 28 & -2.0 & 0 & .009 & -1.011 & 0 & & & \\
\hline 35 & & 0.2 & .007 & -1.010 & -0.004 & & & \\
\hline 46 & -- & .4 & .003 & -1.007 & -.007 & & & \\
\hline 70 &.- & .6 & -.003 & -1.004 & -.007 & & & \\
\hline 93 & $-\ldots$ & .7 & -.005 & -1.003 & -.007 & & & \\
\hline 100 & -- & .8 & -.007 & -1.001 & -.005 & & & \\
\hline 100 & & .9 & -.008 & -1.000 & -.003 & & & \\
\hline 15 & -- & 1.0 & -.008 & -1.0 & 0 & & & \\
\hline 28 & -1.5 & 0 & 0.026 & -1.018 & 0 & 0.027 & -1.031 & 0 \\
\hline 35 & & 0.2 & .022 & -1.017 & -0.010 & .018 & -1.029 & -0.010 \\
\hline 46 & & .4 & .011 & -1.013 & -.017 & -.004 & -1.023 & -.017 \\
\hline 70 & & .6 & -.006 & -1.008 & -.019 & -.033 & -1.014 & -.019 \\
\hline 93 & $\ldots$ & .7 & -.015 & -1.005 & -.017 & -.046 & -1.009 & -.017 \\
\hline 100 & & .8 & -.023 & -1.003 & -.014 & -.054 & -1.005 & -.014 \\
\hline 100 & & .9 & -.031 & -1.001 & -.008 & -.055 & -1.001 & -.008 \\
\hline 15 & - & 1. 0 & -.036 & -1.0 & 0 & -.046 & -1.0 & 0 \\
\hline 28 & -1.0 & 0 & .056 & -0.999 & 0 & .064 & -1.034 & 0 \\
\hline 35 & & 0.2 & .050 & -1.001 & -0.012 & .053 & -1.033 & -0.019 \\
\hline 46 & & .4 & .030 & -1.004 & -.023 & .020 & -1.029 & -.036 \\
\hline 70 & & .6 & -.005 & -1.005 & -.030 & -.032 & -1.021 & -.046 \\
\hline 93 & $-\ldots$ & .7 & -.028 & -1.005 & -.030 & -.062 & -1.015 & -.046 \\
\hline 100 & -- & .8 & -.054 & -1.003 & -.026 & -.092 & -1.008 & -.039 \\
\hline 100 & & .9 & -.081 & -1.001 & -.016 & -.119 & -1.002 & -.025 \\
\hline 15 & & 1. 0 & -.106 & -1.0 & 0 & -.137 & -1.0 & 0 \\
\hline 28 & -0.75 & 0 & 0.066 & -0.954 & 0 & 0.087 & -1.007 & 0 \\
\hline 35 & & 0.2 & .061 & -.960 & -0.003 & .077 & -1.009 & -0.018 \\
\hline 46 & & .4 & .044 & -.975 & -.011 & .043 & -1.014 & -.038 \\
\hline 70 & - & .6 & .004 & -.992 & -.023 & -.022 & -1.015 & -.056 \\
\hline 93 & & .7 & -.028 & -.998 & -.028 & -.067 & -1.013 & -.060 \\
\hline 100 & & .8 & -.068 & -1.000 & -.028 & -.119 & -1.009 & -.055 \\
\hline 100 & & .9 & -.115 & -1.001 & -.020 & -.176 & -1.003 & -.037 \\
\hline 15 & & 1. 0 & -.163 & -1.0 & 0 & -.230 & -1.0 & -.001 \\
\hline 28 & -0.5 & 0 & .056 & -0.857 & 0 & .103 & -0.921 & 0 \\
\hline 35 & - & 0.2 & .057 & -.869 & 0.018 & .096 & -.928 & -0.006 \\
\hline 46 & & .4 & .053 & -.902 & .026 & .069 & -.950 & -.019 \\
\hline 70 & & .6 & .027 & -.950 & .014 & .003 & -.980 & -.042 \\
\hline 93 & & .7 & -.007 & -.974 & 0 & -.056 & -.994 & -.054 \\
\hline 100 & & .8 & -.063 & -.991 & -0.013 & -.138 & -1.002 & -.059 \\
\hline 100 & & .9 & -.141 & -.999 & -.017 & -.244 & -1.003 & -.047 \\
\hline 15 & & 1. 0 & -.235 & -1.0 & 0 & -.365 & -1.0 & -.003 \\
\hline 28 & -0.25 & 0 & 0.012 & -0.695 & 0 & 0.096 & -0.746 & 0 \\
\hline 35 & & 0.2 & .018 & -.707 & 0.050 & .093 & -.755 & 0.016 \\
\hline 46 & & .4 & .035 & -.745 & .092 & .077 & -.787 & .029 \\
\hline 70 & & .6 & .052 & -.821 & .110 & .032 & -.850 & .031 \\
\hline 93 & & .7 & .045 & -.877 & .099 & -.016 & -.897 & .020 \\
\hline 100 & & .8 & .005 & -.940 & .064 & -.106 & -.952 & -.003 \\
\hline 100 & & .9 & -.108 & -.988 & .014 & -.274 & -.993 & -.031 \\
\hline 15 & $\ldots$ & 1.0 & -.313 & -1.0 & 0 & -.539 & -1.0 & -.008 \\
\hline
\end{tabular}

Table 5. Stresses in plate for case 4-Continued

\begin{tabular}{|c|c|c|c|c|c|c|c|c|}
\hline \multirow{2}{*}{$\begin{array}{l}\text { No. of } \\
\text { terms, } \\
\quad m\end{array}$} & \multirow{2}{*}{$\frac{x-a}{c}$} & \multirow{2}{*}{$y$} & \multicolumn{3}{|c|}{704 Results } & \multicolumn{3}{|c|}{ Corrected } \\
\hline & & & $\sigma_{x} / q$ & $\sigma_{y} / q$ & $\tau_{x y} / q$ & $\sigma_{x} / q$ & $\sigma_{y} / q$ & $\tau_{x y} / q$ \\
\hline 28 & 0 & 0 & -.064 & -0.489 & 0 & .063 & -0.485 & 0 \\
\hline 35 & & 0.2 & -.057 & -.488 & 0.066 & .060 & -.488 & 0.034 \\
\hline 46 & & .4 & -.033 & -.488 & .133 & .045 & -.495 & .068 \\
\hline 70 & & .6 & .008 & -.490 & .200 & -.001 & -.502 & .110 \\
\hline 93 & & .7 & 033 & -.493 & .233 & -.039 & -.504 & .140 \\
\hline 100 & & .8 & .061 & -.496 & .263 & -.091 & -.505 & .180 \\
\hline 100 & & .9 & .089 & -.499 & 291 & -.156 & -.504 & .231 \\
\hline 15 & & 1. 0 & $\left\{\begin{array}{r}-.387 \\
.613\end{array}\right.$ & $\left\{\begin{array}{c}-1.0 \\
0\end{array}\right.$ & 0 & $\left\{\begin{array}{r}-.735 \\
.265\end{array}\right.$ & $\left\{\begin{array}{c}-1.0 \\
0\end{array}\right.$ & -.018 \\
\hline 28 & 0.25 & 0 & -0.138 & -0.311 & 0 & 0.019 & -0.198 & 0 \\
\hline 35 & & 0.2 & -.131 & -.296 & 0.045 & .021 & -.193 & 0.030 \\
\hline 46 & & .4 & -.105 & -.250 & .089 & .012 & -.174 & .049 \\
\hline 70 & & .6 & -.043 & -.169 & .116 & -.028 & -.128 & .047 \\
\hline 93 & & .7 & .017 & -.113 & .110 & -.055 & -.089 & .033 \\
\hline 100 & & .8 & .117 & -.054 & .079 & -.065 & -.043 & .002 \\
\hline 100 & & .9 & .295 & -.009 & .025 & -.022 & -.007 & -.036 \\
\hline 15 & & 1. 0 & .561 & 0 & 0 & .091 & 0 & -.029 \\
\hline 28 & 0.5 & 0 & -.167 & -0.241 & 0 & -.008 & -0.006 & 0 \\
\hline 35 & - n. & 0.2 & -.160 & -.221 & 0 & .002 & .023 & 0 \\
\hline 46 & & .4 & -.129 & -.164 & 0 & .012 & .085 & 0 \\
\hline 70 & & .6 & -.040 & -.080 & 0 & -.002 & . 126 & 0 \\
\hline 93 & 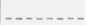 & .7 & .047 & -.040 & 0 & -.012 & .122 & 0 \\
\hline 100 & & .8 & .175 & -.012 & 0 & -.013 & .094 & 0 \\
\hline 100 & & .9 & .346 & 0 & 0 & -.002 & .048 & 0 \\
\hline 15 & & 1. 0 & .541 & 0 & 0 & .011 & 0 & 0 \\
\hline
\end{tabular}

Table 6. Stresses in plate for case 5

$\frac{\text { Loaded length }}{\text { Width }}, \frac{a}{c}=2.0 ; l>>c$

\begin{tabular}{|c|c|c|c|c|c|}
\hline $\begin{array}{c}\text { No. of } \\
\text { terms, } m\end{array}$ & $\frac{x-a}{c}$ & $\frac{y}{c}$ & $\sigma_{x} / q$ & $\sigma_{y} / q$ & $\tau_{x y} / q$ \\
\hline $\begin{array}{r}35 \\
44 \\
59 \\
89 \\
100 \\
100 \\
20\end{array}$ & -2.0 & $\begin{array}{r}0 \\
0.2 \\
.4 \\
.6 \\
.8 \\
.9 \\
1.0\end{array}$ & $\begin{array}{r}0.017 \\
.014 \\
.005 \\
-.005 \\
-.014 \\
-.016 \\
-.015\end{array}$ & $\begin{array}{l}-1.023 \\
-1.021 \\
-1.016 \\
-1.009 \\
-1.003 \\
-1.001 \\
-1.0\end{array}$ & $\begin{array}{l}0 \\
0 \\
0 \\
0 \\
0 \\
0 \\
0\end{array}$ \\
\hline $\begin{array}{r}35 \\
44 \\
59 \\
89 \\
100 \\
100 \\
20\end{array}$ & -1.5 & $\begin{array}{r}0 \\
0.2 \\
.4 \\
.6 \\
.8 \\
.9 \\
1.0\end{array}$ & $\begin{array}{r}.029 \\
.025 \\
-.011 \\
-.007 \\
-.026 \\
-.033 \\
-.036\end{array}$ & $\begin{array}{l}-1.026 \\
-1.024 \\
-1.019 \\
-1.011 \\
-1.004 \\
-1.001 \\
-1.0\end{array}$ & $\begin{array}{l}0 \\
-0.010 \\
-.017 \\
-.019 \\
=.014 \\
-.008 \\
0\end{array}$ \\
\hline $\begin{array}{r}35 \\
44 \\
59 \\
89 \\
100 \\
100 \\
20\end{array}$ & $\begin{array}{c}-1.0 \\
-0 \\
\end{array}$ & $\begin{array}{r}0 \\
0.2 \\
.4 \\
.6 \\
.8 \\
.9 \\
1.0\end{array}$ & $\begin{array}{r}0.064 \\
.056 \\
.033 \\
-.008 \\
-.061 \\
-.088 \\
-.112\end{array}$ & $\begin{array}{l}-1.012 \\
-1.013 \\
-1.013 \\
-1.010 \\
-1.004 \\
-1.001 \\
-1.0\end{array}$ & $\begin{array}{l}0 \\
-0.016 \\
-.030 \\
-.038 \\
-.031 \\
-.019 \\
0\end{array}$ \\
\hline $\begin{array}{r}35 \\
44 \\
59 \\
89 \\
100 \\
100 \\
20\end{array}$ & $\begin{array}{c}-0.75 \\
\end{array}$ & $\begin{array}{r}0 \\
0.2 \\
.4 \\
.6 \\
.8 \\
.9 \\
1.0\end{array}$ & $\begin{array}{r}.081 \\
.074 \\
.050 \\
0 \\
-0.082 \\
-.131 \\
-.180\end{array}$ & $\begin{array}{r}-0.971 \\
-.976 \\
-.987 \\
-.999 \\
-1.003 \\
-1.002 \\
-1.0\end{array}$ & $\begin{array}{l}0 \\
-0.010 \\
-.023 \\
-.037 \\
-.037 \\
-.025 \\
0\end{array}$ \\
\hline $\begin{array}{r}35 \\
44 \\
59 \\
89 \\
100 \\
100 \\
20\end{array}$ & $\begin{array}{c}-0.5 \\
\end{array}$ & $\begin{array}{r}0 \\
0.2 \\
.4 \\
.6 \\
.8 \\
.9 \\
1.0\end{array}$ & $\begin{array}{r}0.083 \\
.080 \\
.064 \\
.020 \\
-.087 \\
-.172 \\
-.270\end{array}$ & $\begin{array}{r}-0.880 \\
-.889 \\
-.918 \\
-.960 \\
-.994 \\
-1.001 \\
-1.0\end{array}$ & $\begin{array}{l}0 \\
0.007 \\
.007 \\
-.007 \\
-.028 \\
-.026 \\
0\end{array}$ \\
\hline $\begin{array}{r}35 \\
44 \\
59 \\
89 \\
100 \\
100 \\
20\end{array}$ & -0.25 & $\begin{array}{l}0 \\
0.2 \\
.4 \\
.6 \\
.8 \\
.9 \\
1.0\end{array}$ & $\begin{array}{r}.056 \\
.056 \\
.055 \\
.043 \\
-.035 \\
-.163 \\
-.379\end{array}$ & $\begin{array}{r}-0.718 \\
-.728 \\
-.763 \\
-.832 \\
-.944 \\
-.990 \\
-1.0\end{array}$ & $\begin{array}{c}0 \\
0.035 \\
.066 \\
.079 \\
.042 \\
-.001 \\
0 .\end{array}$ \\
\hline $\begin{array}{r}35 \\
44 \\
59 \\
89 \\
100 \\
100\end{array}$ & - & $\begin{array}{l}0 \\
0.2 \\
.4 \\
.6 \\
.8 \\
.9\end{array}$ & $\begin{array}{l}0 \\
0 \\
0 \\
0 \\
0 \\
0\end{array}$ & $\begin{array}{r}-0.500 \\
-.500 \\
-.500 \\
-.500 \\
-.500 \\
-.500\end{array}$ & $\begin{array}{l}0 \\
0.050 \\
.103 \\
.162 \\
.232 \\
.269\end{array}$ \\
\hline 20 & & 1.0 & $\left\{\begin{array}{r}-0.500 \\
.500\end{array}\right.$ & $\left\{\begin{array}{r}-1 \\
0\end{array}\right.$ & 0 \\
\hline
\end{tabular}


TABLE 6. Stresses in plate for case 5-Continued

\begin{tabular}{|c|c|c|c|c|c|}
\hline $\begin{array}{l}\text { No. of } \\
\text { terms, } m\end{array}$ & $\frac{x-a}{c}$ & $\frac{y}{c}$ & $\sigma_{x} / q$ & $\sigma_{y} / q$ & $\tau_{x y} / q$ \\
\hline $\begin{array}{r}35 \\
44 \\
59 \\
89 \\
100 \\
100 \\
20\end{array}$ & $\begin{array}{c}0.25 \\
\end{array}$ & $\begin{array}{r}0 \\
0.2 \\
.4 \\
.6 \\
.8 \\
.9 \\
1.0\end{array}$ & $\begin{array}{r}-.056 \\
-.056 \\
-.055 \\
-.043 \\
.035 \\
.163 \\
.380\end{array}$ & $\begin{array}{l}-.282 \\
-.272 \\
-.237 \\
-.168 \\
-.056 \\
-.010 \\
0\end{array}$ & $\begin{array}{r}0 \\
0.035 \\
.066 \\
.080 \\
.042 \\
-.001 \\
0\end{array}$ \\
\hline $\begin{array}{r}35 \\
44 \\
59 \\
89 \\
100 \\
100 \\
20\end{array}$ & $\begin{array}{c}0.5 \\
\\
\end{array}$ & $\begin{array}{r}0 \\
0.2 \\
.4 \\
.6 \\
.8 \\
.9 \\
1.0\end{array}$ & $\begin{array}{r}-0.084 \\
-.080 \\
-.064 \\
-.020 \\
.088 \\
.173 \\
.271\end{array}$ & $\begin{array}{r}-0.121 \\
-.111 \\
-.082 \\
-.040 \\
-.006 \\
.001 \\
0\end{array}$ & $\begin{array}{l}0 \\
0.007 \\
-.007 \\
-.007 \\
-.028 \\
-.026 \\
0\end{array}$ \\
\hline $\begin{array}{r}35 \\
44 \\
59 \\
89 \\
100 \\
100 \\
20\end{array}$ & $\begin{array}{c}0.75 \\
\\
\\
\end{array}$ & $\begin{array}{r}0 \\
0.2 \\
.4 \\
.6 \\
.8 \\
.9 \\
1.0\end{array}$ & $\begin{array}{c}-.082 \\
-.074 \\
-.050 \\
0 \\
0.082 \\
.131 \\
.181\end{array}$ & $\begin{array}{r}-0.029 \\
-.025 \\
-.013 \\
-.001 \\
.003 \\
.002 \\
0\end{array}$ & $\begin{array}{r}0 \\
-0.010 \\
-.023 \\
-.037 \\
-.037 \\
-.024 \\
0\end{array}$ \\
\hline $\begin{array}{r}35 \\
44 \\
59 \\
89 \\
100 \\
100 \\
20\end{array}$ & $\begin{array}{c}1.0 \\
-\end{array}$ & $\begin{array}{r}0 \\
0.2 \\
.4 \\
.6 \\
.8 \\
.9 \\
1.0\end{array}$ & $\begin{array}{r}-0.064 \\
-.057 \\
-.033 \\
.008 \\
.061 \\
.089 \\
.113\end{array}$ & $\begin{array}{r}0.011 \\
.012 \\
.012 \\
.010 \\
.004 \\
.001 \\
0\end{array}$ & $\begin{array}{c}0 \\
-0.016 \\
-.030 \\
-.038 \\
-.031 \\
-.019 \\
0\end{array}$ \\
\hline $\begin{array}{r}35 \\
44 \\
59 \\
89 \\
100 \\
100 \\
20\end{array}$ & $\begin{array}{c}2.0 \\
\end{array}$ & $\begin{array}{r}0 \\
0.2 \\
.4 \\
.6 \\
.8 \\
.9 \\
1.0\end{array}$ & $\begin{array}{r}-.008 \\
-.007 \\
-.003 \\
.003 \\
.007 \\
.008 \\
.008\end{array}$ & $\begin{array}{l}0.012 \\
.011 \\
.008 \\
.005 \\
.001 \\
.001 \\
0 .\end{array}$ & $\begin{aligned} & 0 \\
&-0.004 \\
&-.007 \\
&-.008 \\
&-.005 \\
&-.003 \\
& 0\end{aligned}$ \\
\hline
\end{tabular}

TABLE 7.-Stresses in plate for case 6 Loaded length, Width $\frac{\mathrm{a}}{c}=1.5 ; l>>c$

\begin{tabular}{|c|c|c|c|c|c|}
\hline $\begin{array}{l}\text { No. of } \\
\text { terms, } m\end{array}$ & $\frac{x-a}{c}$ & $\frac{y}{c}$ & $\sigma_{x} / q$ & $\sigma_{y} / q$ & $\tau_{x y} / q$ \\
\hline $\begin{array}{r}35 \\
44 \\
59 \\
89 \\
100 \\
100 \\
20\end{array}$ & $\begin{array}{c}-1.5 \\
\end{array}$ & $\begin{array}{r}0 \\
0.2 \\
.4 \\
.6 \\
.8 \\
.9 \\
1.0\end{array}$ & $\begin{array}{r}0.056 \\
.047 \\
.022 \\
-.013 \\
-.049 \\
-.063 \\
-.072\end{array}$ & $\begin{array}{l}-1.044 \\
-1.041 \\
-1.032 \\
-1.019 \\
-1.006 \\
-1.002 \\
-1.0\end{array}$ & $\begin{array}{l}0 \\
0 \\
0 \\
0 \\
0 \\
0 \\
0\end{array}$ \\
\hline $\begin{array}{r}35 \\
44 \\
59 \\
89 \\
100 \\
100 \\
20\end{array}$ & $\begin{array}{c}-1.0 \\
\end{array}$ & $\begin{array}{r}0 \\
0.2 \\
.4 \\
.6 \\
.8 \\
.9 \\
1.0\end{array}$ & $\begin{array}{r}.073 \\
.063 \\
.036 \\
-.010 \\
-.069 \\
-.097 \\
-.121\end{array}$ & $\begin{array}{l}-1.023 \\
-1.022 \\
-1.020 \\
-1.015 \\
-1.006 \\
-1.002 \\
-1.0\end{array}$ & $\begin{array}{l}0 \\
-0.012 \\
-.023 \\
-.030 \\
=.026 \\
-.016 \\
0\end{array}$ \\
\hline $\begin{array}{r}35 \\
44 \\
59 \\
89 \\
100 \\
100 \\
20\end{array}$ & -0.75 & $\begin{array}{r}0 \\
0.2 \\
.4 \\
.6 \\
.8 \\
.9 \\
1.0\end{array}$ & $\begin{array}{r}0.086 \\
.078 \\
.051 \\
-.002 \\
-.085 \\
-.135 \\
-.183\end{array}$ & $\begin{array}{l}-0.978 \\
-.982 \\
-.992 \\
-1.002 \\
-1.003 \\
-1.002 \\
-1.0\end{array}$ & $\begin{array}{c}0 \\
-0.008 \\
-.019 \\
-.032 \\
-.034 \\
-.023 \\
0\end{array}$ \\
\hline $\begin{array}{r}35 \\
44 \\
59 \\
89 \\
100 \\
100 \\
20\end{array}$ & -0.5 & $\begin{array}{r}0 \\
0.2 \\
.4 \\
.6 \\
.8 \\
.9 \\
1.0\end{array}$ & $\begin{array}{r}.085 \\
.081 \\
.065 \\
.019 \\
-.039 \\
-.173 \\
-.271\end{array}$ & $\begin{array}{l}-0.884 \\
-.893 \\
-.921 \\
-.962 \\
-.995 \\
-1.001 \\
-1.0\end{array}$ & $\begin{array}{l}0 \\
0.009 \\
-.009 \\
-.005 \\
-.027 \\
-.025 \\
0\end{array}$ \\
\hline $\begin{array}{r}35 \\
44 \\
59 \\
89 \\
100 \\
100 \\
20\end{array}$ & -0.25 & $\begin{array}{r}0 \\
0.2 \\
.4 \\
.6 \\
.8 \\
.9 \\
1.0\end{array}$ & $\begin{array}{r}0.056 \\
.056 \\
.055 \\
.043 \\
-.036 \\
-.163 \\
-.379\end{array}$ & $\begin{array}{l}-0.720 \\
-.730 \\
-.764 \\
-.833 \\
-.944 \\
-.990 \\
-1.0\end{array}$ & $\begin{array}{l}0 . \\
0.036 \\
.067 \\
.080 \\
.042 \\
-.001 \\
0\end{array}$ \\
\hline $\begin{array}{r}35 \\
44 \\
59 \\
89 \\
100 \\
100\end{array}$ & 0 & $\begin{array}{l}0 \\
0.2 \\
.4 \\
.6 \\
.8 \\
.9\end{array}$ & $\begin{array}{l}0 \\
0 \\
0 \\
0 \\
0 \\
0.001\end{array}$ & $\begin{array}{l}-0.501 \\
-.501 \\
-.501 \\
-.500 \\
-.500 \\
-.500\end{array}$ & $\begin{array}{l}0 \\
0.050 \\
.103 \\
.163 \\
.232 \\
.269\end{array}$ \\
\hline 20 & . & 1.0 & $\left\{\begin{array}{r}-.499 \\
.501\end{array}\right.$ & $\left\{\begin{array}{c}-1.0 \\
0\end{array}\right.$ & 0 \\
\hline
\end{tabular}

TABLe 7.-Stresses in plate for case 6-Continued

\begin{tabular}{|c|c|c|c|c|c|}
\hline $\begin{array}{l}\text { No. of } \\
\text { terms, } m\end{array}$ & $\frac{x-a}{c}$ & $\frac{y}{c}$ & $\sigma_{x} / q$ & $\sigma_{y} / q$ & $\tau_{x y} / q$ \\
\hline $\begin{array}{r}35 \\
44 \\
59 \\
89 \\
100 \\
100 \\
20\end{array}$ & $\begin{array}{c}0.25 \\
\end{array}$ & $\begin{array}{r}0 \\
0.2 \\
.4 \\
.6 \\
.8 \\
.9 \\
1.0\end{array}$ & $\begin{array}{r}-0.056 \\
-.056 \\
-.056 \\
-.043 \\
.026 \\
.164 \\
.380\end{array}$ & $\begin{array}{r}-0.282 \\
-.272 \\
-.238 \\
-.168 \\
-.056 \\
-.010 \\
0\end{array}$ & $\begin{array}{r}0 \\
0.035 \\
.066 \\
.080 \\
.042 \\
-.001 \\
0\end{array}$ \\
\hline $\begin{array}{r}35 \\
44 \\
59 \\
89 \\
100 \\
100 \\
20\end{array}$ & $\begin{array}{c}0.5 \\
\\
\end{array}$ & $\begin{array}{r}0 \\
0.2 \\
.4 \\
.6 \\
.8 \\
.9 \\
1.0\end{array}$ & $\begin{array}{r}-.084 \\
-.080 \\
-.065 \\
-.020 \\
.088 \\
.173 \\
.271\end{array}$ & $\begin{array}{r}-0.121 \\
-.111 \\
-.082 \\
-.040 \\
-.006 \\
.001 \\
0\end{array}$ & $\begin{array}{l}0 \\
0.007 \\
.007 \\
-.007 \\
-.028 \\
-.026 \\
0\end{array}$ \\
\hline $\begin{array}{r}35 \\
44 \\
59 \\
89 \\
100 \\
100 \\
20\end{array}$ & $\begin{array}{c}0.75 \\
- \\
\end{array}$ & $\begin{array}{r}0 \\
0.2 \\
.4 \\
.6 \\
.8 \\
.9 \\
1.0\end{array}$ & $\begin{array}{r}-0.082 \\
-.075 \\
-.050 \\
0 \\
0.082 \\
.132 \\
.181\end{array}$ & $\begin{array}{r}-0.029 \\
-.025 \\
-.013 \\
-.001 \\
.003 \\
.002 \\
0\end{array}$ & $\begin{array}{c}0 \\
-0.010 \\
-.023 \\
-.037 \\
-.037 \\
-.024 \\
0\end{array}$ \\
\hline $\begin{array}{r}35 \\
44 \\
59 \\
89 \\
100 \\
100 \\
20\end{array}$ & $\begin{array}{c}1.0 \\
\\
\end{array}$ & $\begin{array}{r}0 \\
0.2 \\
.4 \\
.6 \\
.8 \\
.9 \\
1.0\end{array}$ & $\begin{array}{r}-.064 \\
-.057 \\
-.033 \\
.008 \\
.061 \\
.089 \\
.114\end{array}$ & $\begin{array}{r}0.011 \\
.012 \\
.012 \\
.010 \\
.004 \\
.001 \\
0\end{array}$ & $\begin{array}{c}0 \\
-0.016 \\
-.030 \\
-.038 \\
-.031 \\
-.019 \\
0\end{array}$ \\
\hline $\begin{array}{r}35 \\
44 \\
59 \\
89 \\
100 \\
100 \\
20\end{array}$ & $\begin{array}{c}1.5 \\
\\
\\
\end{array}$ & $\begin{array}{r}0 \\
0.2 \\
.4 \\
.6 \\
.8 \\
.9 \\
1.0\end{array}$ & $\begin{array}{r}-0.028 \\
-.023 \\
-.011 \\
.006 \\
.025 \\
.032 \\
.036\end{array}$ & $\begin{array}{r}0.022 \\
.021 \\
.016 \\
.010 \\
.003 \\
.001 \\
0\end{array}$ & $\begin{array}{l}0 \\
-0.011 \\
-.019 \\
-.021 \\
-.015 \\
-.009 \\
0\end{array}$ \\
\hline $\begin{array}{r}35 \\
44 \\
59 \\
89 \\
100 \\
100 \\
20\end{array}$ & $\begin{array}{r}2.5 \\
- \\
\end{array}$ & $\begin{array}{r}0 \\
0.2 \\
.4 \\
.6 \\
.8 \\
.9 \\
1.0\end{array}$ & $\begin{array}{l}-.002 \\
-.001 \\
0 \\
0.001 \\
.001 \\
.001 \\
0\end{array}$ & $\begin{array}{l}0.004 \\
.004 \\
.003 \\
.002 \\
0 \\
0 \\
0\end{array}$ & $\begin{array}{l}0 \\
-0.001 \\
-.002 \\
-.002 \\
-.001 \\
-.001 \\
0\end{array}$ \\
\hline
\end{tabular}

Table 8. Stresses in plate for case 7 $\frac{\text { Loaded length }}{\text { Width }}, \frac{a}{c}=1.0 ; l>>c$

\begin{tabular}{|c|c|c|c|c|c|}
\hline $\begin{array}{c}\text { No. of } \\
\text { terms, } m\end{array}$ & $\frac{x-a}{c}$ & $\frac{y}{c}$ & $\sigma_{x} / q$ & $\sigma_{y} / q$ & $\tau_{x y} / q$ \\
\hline $\begin{array}{r}35 \\
44 \\
59 \\
89 \\
100 \\
100 \\
20\end{array}$ & $\begin{array}{c}-1.0 \\
\end{array}$ & $\begin{array}{r}0 \\
0.2 \\
.4 \\
.6 \\
.8 \\
.9 \\
1.0\end{array}$ & $\begin{array}{r}0.129 \\
.113 \\
.066 \\
-.015 \\
-.122 \\
-.178 \\
-.227\end{array}$ & $\begin{array}{l}-1.022 \\
-1.023 \\
-1.024 \\
-1.020 \\
-1.009 \\
-1.002 \\
-1.0\end{array}$ & $\begin{array}{l}0 \\
0 \\
0 \\
0 \\
0 \\
0 \\
0\end{array}$ \\
\hline $\begin{array}{r}35 \\
44 \\
59 \\
89 \\
100 \\
100 \\
20\end{array}$ & $\begin{array}{c}-0.75 \\
\end{array}$ & $\begin{array}{r}0 \\
0.2 \\
.4 \\
.6 \\
.8 \\
.9 \\
1.0\end{array}$ & $\begin{array}{r}.126 \\
.112 \\
.070 \\
-.008 \\
-.123 \\
-.187 \\
-.247\end{array}$ & $\begin{array}{r}-0.994 \\
-.997 \\
-1.005 \\
-1.010 \\
-1.007 \\
-1.002 \\
-1.0\end{array}$ & $\begin{array}{l}0 \\
0.004 \\
-.003 \\
-.006 \\
-.014 \\
-.011 \\
0\end{array}$ \\
\hline $\begin{array}{r}35 \\
44 \\
59 \\
89 \\
100 \\
100 \\
20\end{array}$ & $\begin{array}{c}-0.5 \\
\end{array}$ & $\begin{array}{r}0 \\
0.2 \\
.4 \\
.6 \\
.8 \\
.9 \\
1.0\end{array}$ & $\begin{array}{r}0.111 \\
.103 \\
.076 \\
.014 \\
-.112 \\
-.204 \\
-.307\end{array}$ & $\begin{array}{r}-0.902 \\
-.910 \\
-.934 \\
-.970 \\
-.997 \\
-1.001 \\
-1.0\end{array}$ & $\begin{array}{r}0 \\
0.018 \\
.026 \\
.014 \\
-.013 \\
-.017 \\
0\end{array}$ \\
\hline $\begin{array}{r}35 \\
44 \\
59 \\
89 \\
100 \\
100 \\
20\end{array}$ & $\begin{array}{c}-0.25 \\
-1 \\
\end{array}$ & $\begin{array}{r}0 \\
0.2 \\
.4 \\
.6 \\
.8 \\
.9 \\
1.0\end{array}$ & $\begin{array}{r}.072 \\
.069 \\
.061 \\
.039 \\
-.049 \\
-.180 \\
-.397\end{array}$ & $\begin{array}{r}-0.735 \\
-.744 \\
-.775 \\
-.839 \\
-.946 \\
-.991 \\
-1.0\end{array}$ & $\begin{array}{l}0 \\
0.042 \\
.078 \\
.093 \\
.051 \\
.004 \\
0\end{array}$ \\
\hline
\end{tabular}


TABLE 8. Stresses in plate for case 7 -Continued

\begin{tabular}{|c|c|c|c|c|c|}
\hline $\begin{array}{l}\text { No. of } \\
\text { terms, } m\end{array}$ & $\frac{x-a}{c}$ & $\frac{y}{c}$ & $\sigma_{x} / q$ & $\sigma_{y} / q$ & $\boldsymbol{\tau}_{x y} / q$ \\
\hline $\begin{array}{r}35 \\
44 \\
59 \\
89 \\
100 \\
100 \\
20\end{array}$ & 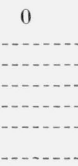 & $\begin{array}{r}0 \\
0.2 \\
.4 \\
.6 \\
.8 \\
.9 \\
1.0\end{array}$ & $\begin{array}{r}0.008 \\
.007 \\
.003 \\
-.003 \\
-.007 \\
-.008 \\
-.508 \\
492\end{array}$ & $\begin{array}{r}-0.512 \\
-.511 \\
-.508 \\
-.505 \\
-.501 \\
-.500 \\
-1.0\end{array}$ & $\begin{array}{l}0 \\
0.055 \\
.111 \\
.170 \\
.237 \\
.272 \\
0\end{array}$ \\
\hline $\begin{array}{r}35 \\
44 \\
59 \\
89 \\
100 \\
100 \\
20\end{array}$ & 0.25 & $\begin{array}{r}0 \\
0.2 \\
.4 \\
.6 \\
.8 \\
.9 \\
1.0\end{array}$ & $\begin{array}{r}-.052 \\
-.053 \\
-.054 \\
-.045 \\
.032 \\
.160 \\
.377\end{array}$ & $\begin{array}{r}-0.289 \\
-.278 \\
-.242 \\
-.170 \\
-.057 \\
-.010 \\
0\end{array}$ & $\begin{array}{l}0 \\
0.038 \\
.070 \\
.084 \\
.044 \\
0 \\
0\end{array}$ \\
\hline $\begin{array}{r}35 \\
44 \\
59 \\
89 \\
100 \\
100 \\
20\end{array}$ & 0.5 & $\begin{array}{r}0 \\
0.2 \\
.4 \\
.6 \\
.8 \\
.9 \\
1.0\end{array}$ & $\begin{array}{r}-0.082 \\
-.079 \\
-.064 \\
-.021 \\
.086 \\
.172 \\
.271\end{array}$ & $\begin{array}{r}-0.125 \\
-.114 \\
-.085 \\
-.041 \\
-.006 \\
.001 \\
0\end{array}$ & $\begin{array}{l}0 \\
0.009 \\
.009 \\
-.005 \\
-.027 \\
-.025 \\
0\end{array}$ \\
\hline $\begin{array}{r}35 \\
44 \\
59 \\
89 \\
100 \\
100 \\
20\end{array}$ & 0.75 & $\begin{array}{r}0 \\
0.2 \\
.4 \\
.6 \\
.8 \\
.9 \\
1.0\end{array}$ & $\begin{array}{c}-.081 \\
-.074 \\
-.050 \\
0 \\
0.082 \\
.132 \\
.182\end{array}$ & $\begin{array}{r}-0.031 \\
-.027 \\
-.014 \\
-.002 \\
.002 \\
.001 \\
0\end{array}$ & $\begin{array}{c}0 \\
-0.010 \\
-.022 \\
-.036 \\
-.037 \\
-.024 \\
0\end{array}$ \\
\hline $\begin{array}{r}35 \\
44 \\
59 \\
89 \\
100 \\
100 \\
20\end{array}$ & 1. 0 & $\begin{array}{r}0 \\
0.2 \\
.4 \\
.6 \\
.8 \\
.9 \\
1.0\end{array}$ & $\begin{array}{r}-0.064 \\
-.057 \\
-.033 \\
.007 \\
.061 \\
.089 \\
.114\end{array}$ & $\begin{array}{l}0.010 \\
.011 \\
.011 \\
.010 \\
.004 \\
.001 \\
0\end{array}$ & $\begin{array}{r}0 \\
-0.016 \\
-.030 \\
-.038 \\
-.031 \\
-.019 \\
0\end{array}$ \\
\hline $\begin{array}{r}35 \\
44 \\
59 \\
89 \\
100 \\
100 \\
20\end{array}$ & 1.5 & $\begin{array}{r}0 \\
0.2 \\
.4 \\
.6 \\
.8 \\
.9 \\
1.0\end{array}$ & $\begin{array}{r}-.028 \\
-.024 \\
-.011 \\
.006 \\
.025 \\
.032 \\
.036\end{array}$ & $\begin{array}{l}0.022 \\
.021 \\
.016 \\
.010 \\
.003 \\
.001 \\
0\end{array}$ & $\begin{array}{c}0 \\
-0.011 \\
-.019 \\
-.021 \\
-.015 \\
-.009 \\
0\end{array}$ \\
\hline $\begin{array}{r}35 \\
44 \\
59 \\
89 \\
100 \\
100 \\
20\end{array}$ & 2.0 & $\begin{array}{r}0 \\
0.2 \\
.4 \\
.6 \\
.8 \\
.9 \\
1.0\end{array}$ & $\begin{array}{r}-0.009 \\
-.007 \\
-.003 \\
.003 \\
.007 \\
.008 \\
.008\end{array}$ & $\begin{array}{l}0.012 \\
.011 \\
.008 \\
.005 \\
.001 \\
0 \\
0\end{array}$ & $\begin{array}{r}0 \\
-0.004 \\
-.007 \\
-.008 \\
-.005 \\
-.003 \\
0\end{array}$ \\
\hline
\end{tabular}

\section{Discussion}

\subsection{Plate With Distributed Load}

The results given herein are directly applicable to a finite plate loaded by uniformly distributed equal loads along portions of two longitudinal edges. The ratio of the length of the loaded portion to the total length of the plate is given in column 5 of table 1 . The ratios of the loaded length of plate to the width and of the unloaded length of plate to the width are given in columns 6 and 7 of table 1 .

A comparison of figures 7 to 9 shows that, in accordance with St. Venant's Principle, the discontinuity in the loading affects the stress distribution in the plate for a distance to either side of the discontinuity equal to the width of the plate. The stresses for case 1 , table 2 , have the same distribution and are of about the same magnitude as those for case 5 , table 6 . It is inferred, therefore, that if the ratios of the loaded length of plate to the width of the plate and of the unloaded length to the width
TABLE 9. Stresses in plate for case 8

\begin{tabular}{|c|c|c|c|c|c|}
\hline $\begin{array}{c}\text { No. of } \\
\text { terms, } m\end{array}$ & $\frac{x-a}{c}$ & $\frac{y}{c}$ & $\sigma_{x} / q$ & $\sigma_{y} / q$ & $\tau_{x y} / q$ \\
\hline $\begin{array}{r}35 \\
44 \\
59 \\
89 \\
100 \\
100 \\
20\end{array}$ & -0.5 & $\begin{array}{r}0 \\
0.2 \\
.4 \\
.6 \\
.8 \\
.9 \\
1.0\end{array}$ & $\begin{array}{r}0.167 \\
.160 \\
.129 \\
.040 \\
-.175 \\
-.345 \\
-.541\end{array}$ & $\begin{array}{l}-0.759 \\
-.779 \\
-.836 \\
-.920 \\
-.988 \\
-1.001 \\
-1.0\end{array}$ & $\begin{array}{l}0 \\
0 \\
0 \\
0 \\
0 \\
0 \\
0\end{array}$ \\
\hline $\begin{array}{r}35 \\
44 \\
59 \\
89 \\
100 \\
100 \\
20\end{array}$ & -0.25 & $\begin{array}{l}0 \\
0.2 \\
.4 \\
.6 \\
.8 \\
.9 \\
1.0\end{array}$ & $\begin{array}{r}.138 \\
.131 \\
.105 \\
.043 \\
-.117 \\
-.294 \\
-.561\end{array}$ & $\begin{array}{c}-0.689 \\
-.704 \\
-.750 \\
-.831 \\
-.946 \\
-.991 \\
-1.0\end{array}$ & $\begin{array}{l}0 \\
0.045 \\
.089 \\
.116 \\
.079 \\
.023 \\
0\end{array}$ \\
\hline $\begin{array}{r}35 \\
44 \\
59 \\
89 \\
100 \\
100 \\
20\end{array}$ & $\begin{array}{ll}- \\
\cdots\end{array}$ & $\begin{array}{l}0 \\
0.2 \\
.4 \\
.6 \\
.8 \\
.9 \\
1.0\end{array}$ & $\begin{array}{r}0.064 \\
.057 \\
.033 \\
-.008 \\
-.061 \\
-.089 \\
-.613 \\
.387\end{array}$ & $\begin{array}{l}-0.511 \\
-.512 \\
-.512 \\
-.510 \\
-.504 \\
-.501 \\
-1.0 \\
0\end{array}$ & $\begin{array}{l}0 \\
0.066 \\
.133 \\
.200 \\
.263 \\
.288 \\
0\end{array}$ \\
\hline $\begin{array}{r}35 \\
44 \\
59 \\
89 \\
100 \\
100 \\
20\end{array}$ & 0.25 & $\begin{array}{r}0 \\
0.2 \\
.4 \\
.6 \\
.8 \\
.9 \\
1.0\end{array}$ & $\begin{array}{r}-.012 \\
-.018 \\
-.035 \\
-.052 \\
-.005 \\
.108 \\
.313\end{array}$ & $\begin{array}{l}-0.305 \\
-.293 \\
-.255 \\
-.179 \\
-.060 \\
-.011 \\
0\end{array}$ & $\begin{array}{l}0 \\
0.050 \\
.092 \\
.110 \\
.064 \\
.012 \\
0\end{array}$ \\
\hline $\begin{array}{r}35 \\
44 \\
59 \\
89 \\
100 \\
100 \\
20\end{array}$ & - & $\begin{array}{l}0 \\
0.2 \\
.4 \\
.6 \\
.8 \\
.9 \\
1.0\end{array}$ & $\begin{array}{r}-0.056 \\
-.056 \\
-.053 \\
-.027 \\
.063 \\
.141 \\
.235\end{array}$ & $\begin{array}{l}-0.143 \\
-.131 \\
-.098 \\
-.050 \\
-.009 \\
0 \\
0\end{array}$ & $\begin{array}{l}0 \\
0.018 \\
.026 \\
.014 \\
-.013 \\
-.017 \\
0\end{array}$ \\
\hline $\begin{array}{r}35 \\
44 \\
59 \\
89 \\
100 \\
100 \\
20\end{array}$ & 0.75 & $\begin{array}{l}0 \\
0.2 \\
.4 \\
.6 \\
.8 \\
.9 \\
1.0\end{array}$ & $\begin{array}{r}-.066 \\
-.061 \\
-.044 \\
-.004 \\
.068 \\
.115 \\
.163\end{array}$ & $\begin{array}{l}-0.046 \\
-.040 \\
-.025 \\
-.008 \\
0 \\
0.001 \\
0\end{array}$ & $\begin{array}{c}0 \\
-0.003 \\
-.011 \\
-.023 \\
-.028 \\
-.019 \\
0\end{array}$ \\
\hline $\begin{array}{r}35 \\
44 \\
59 \\
89 \\
100 \\
100 \\
20\end{array}$ & 1.0 & $\begin{array}{r}0 \\
0.2 \\
.4 \\
.6 \\
.8 \\
.9 \\
1.0\end{array}$ & $\begin{array}{r}-0.056 \\
-.050 \\
-.030 \\
.005 \\
.054 \\
.081 \\
.106\end{array}$ & $\begin{array}{r}-0.001 \\
.001 \\
.004 \\
.005 \\
.003 \\
.001 \\
0\end{array}$ & $\begin{array}{c}0 \\
-0.012 \\
-.023 \\
-.030 \\
-.026 \\
-.016 \\
0\end{array}$ \\
\hline $\begin{array}{r}35 \\
44 \\
59 \\
89 \\
100 \\
100 \\
20\end{array}$ & 1. 5 & $\begin{array}{r}0 \\
0.2 \\
.4 \\
.6 \\
.8 \\
.9 \\
1.0\end{array}$ & $\begin{array}{r}-.026 \\
-.022 \\
-.011 \\
.006 \\
.023 \\
.031 \\
.036\end{array}$ & $\begin{array}{l}0.018 \\
.017 \\
.013 \\
.008 \\
.003 \\
.001 \\
0\end{array}$ & $\begin{array}{c}0 \\
-0.010 \\
-.017 \\
-.019 \\
-.014 \\
-.008 \\
0\end{array}$ \\
\hline $\begin{array}{r}35 \\
44 \\
59 \\
89 \\
100 \\
100 \\
20\end{array}$ & 2.5 & $\begin{array}{r}0 \\
0.2 \\
.4 \\
.6 \\
.8 \\
.9 \\
1.0\end{array}$ & $\begin{array}{r}-0.002 \\
-.001 \\
0 \\
0.001 \\
.001 \\
.001 \\
.001\end{array}$ & $\begin{array}{l}0.004 \\
.004 \\
.003 \\
.001 \\
0 \\
0 \\
0\end{array}$ & $\begin{array}{c}0 \\
-0.001 \\
-.002 \\
-.002 \\
-.001 \\
-.001 \\
0\end{array}$ \\
\hline
\end{tabular}

are greater than 2.0 , the stress distributions will be similar to and the stresses will be of the some magnitude as those shown in figure 8 and listed in tables 2 and 6 .

For those points in the plate at a distance twice the width of the plate from the boundary of the load, the stresses in the direction of loading, $\sigma_{y} / q$, will be either 1.0 or 0 , depending upon whether the points are in the loaded or unloaded parts of the plate, respectively. The stresses transverse to the direction of loading, $\sigma_{x} / q$, and the shear stresses, $\tau_{x y} / q$, will be zero. 
The stresses in the direction of loading are, at the most, only 4 percent higher than the applied load. However when the loading is over only a small portion of the plate length, case 8 , the stresses decrease from values equal to the applied load at the boundary to values equal to only about 76 percent of the applied load at the center, figure $9 \mathrm{a}$.

The stresses normal to the direction of loading, $\sigma_{x} / q$, are of the same order of magnitude and have the same distribution but are of opposite sign on either side of the discontinuity for cases 1 and 5 (see figure $8 b)$. The distribution of the stresses changes somewhat as the ratio of loaded length of plate to width of plate is decreased from 2.0 to 1.0 , cases 5 to 7 , but the maximum stress does not. For case 8 where the ratio of loaded portion of the plate to width of the plate is 0.5 , there is a buildup of stresses in the loaded portion of the plate near the discontinuity in the loading, figure $9 \mathrm{~b}$. This same increase in $\sigma_{x} / q$, is found in the loaded portion of the plate as the ratio of nonloaded length to width of the plate is decreased from 2.0 to 0.5 , being most significant when the ratio is smallest, case 4 , figure $7 \mathrm{~b}$. The distribution of the axial stresses across the width of the plate for case 8 is shown in figure 10 .

The shearing stresses increase to almost 30 percent of the applied load near the boundary of the plate in the region of the discontinuity in the loading,

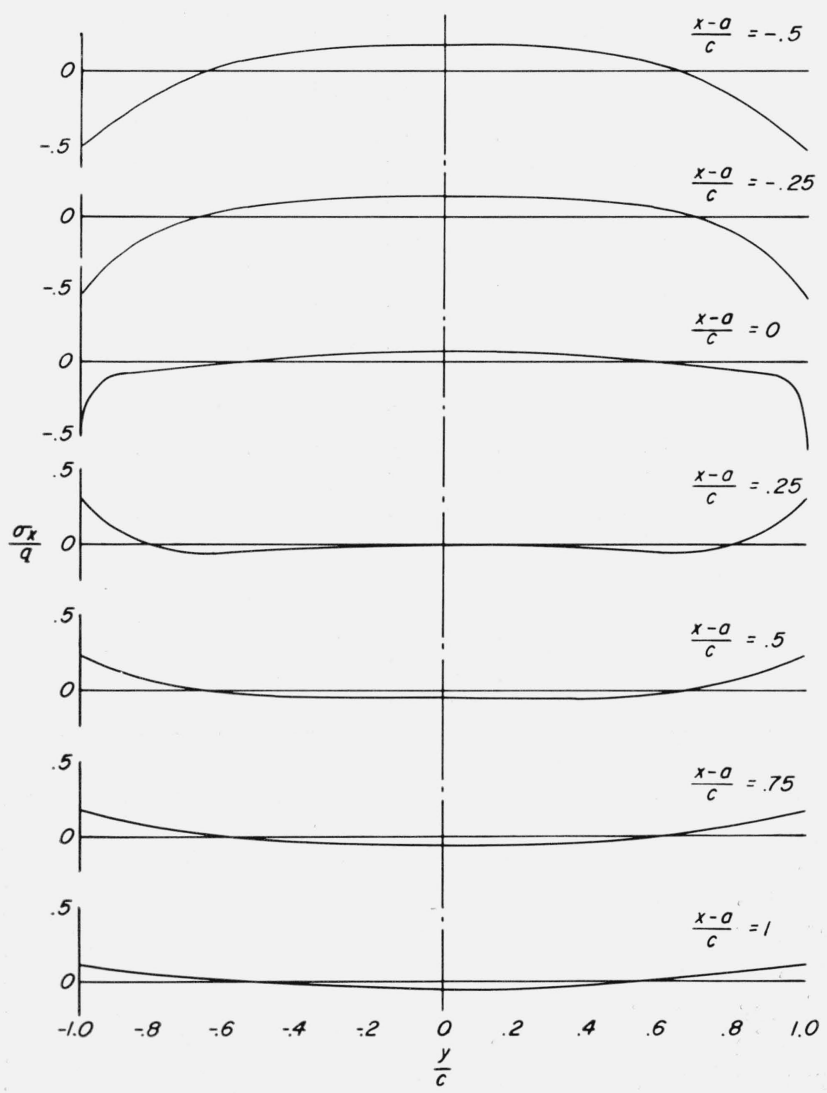

Figure 10. Distribution of axial stresses across_width of plate for case 8. figures $7 \mathrm{c}$ to $9 \mathrm{c}$. These stresses should approach a theoretical value of $\pm 1 / \pi$ as the edge of the plate is approached and then go to zero on the boundary. In other regions of the plate, the shearing stresses are insignificant.

\subsection{Comparison With Previously Published Results}

Barton [8] investigated the problem of a long circular cylinder loaded with a band of uniform pressure over a finite length. His figures 19 and 20 for a loading length of one diameter and figures 22 and 23 for a loading length of one-half diameter are of particular interest. These load lengths are similar to cases 7 and 8 of this paper, with a loading length equal to the width of plate and one-half of the width, respectively. Barton's intermediate values of stresses differ considerably from the values shown in figures 8 and 9 , but the general patterns and extreme values are strikingly similar. Considering the difference in geometries between these problems, this general similarity between the two sets of results is remarkable.

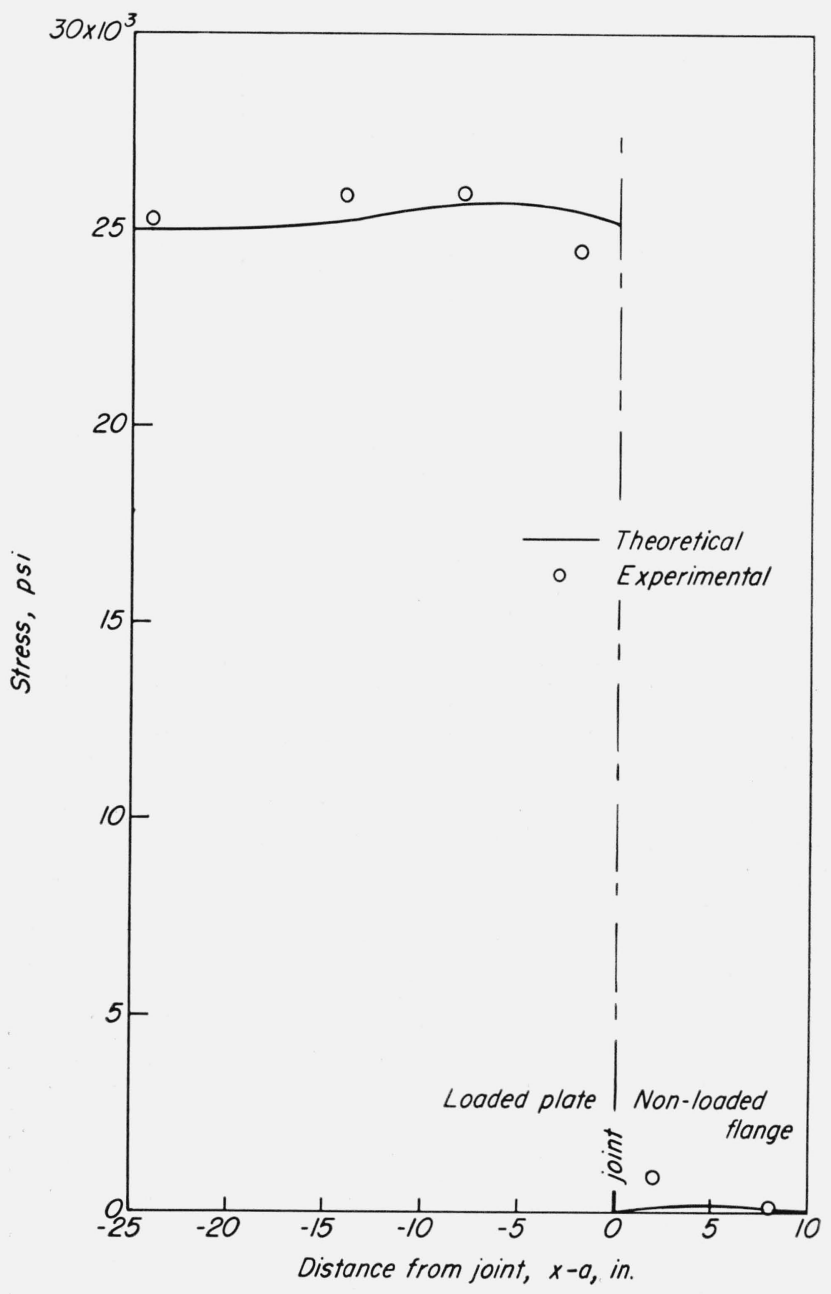

FIGURE 11. Stresses in longitudinal direction along center line of welded joint. 

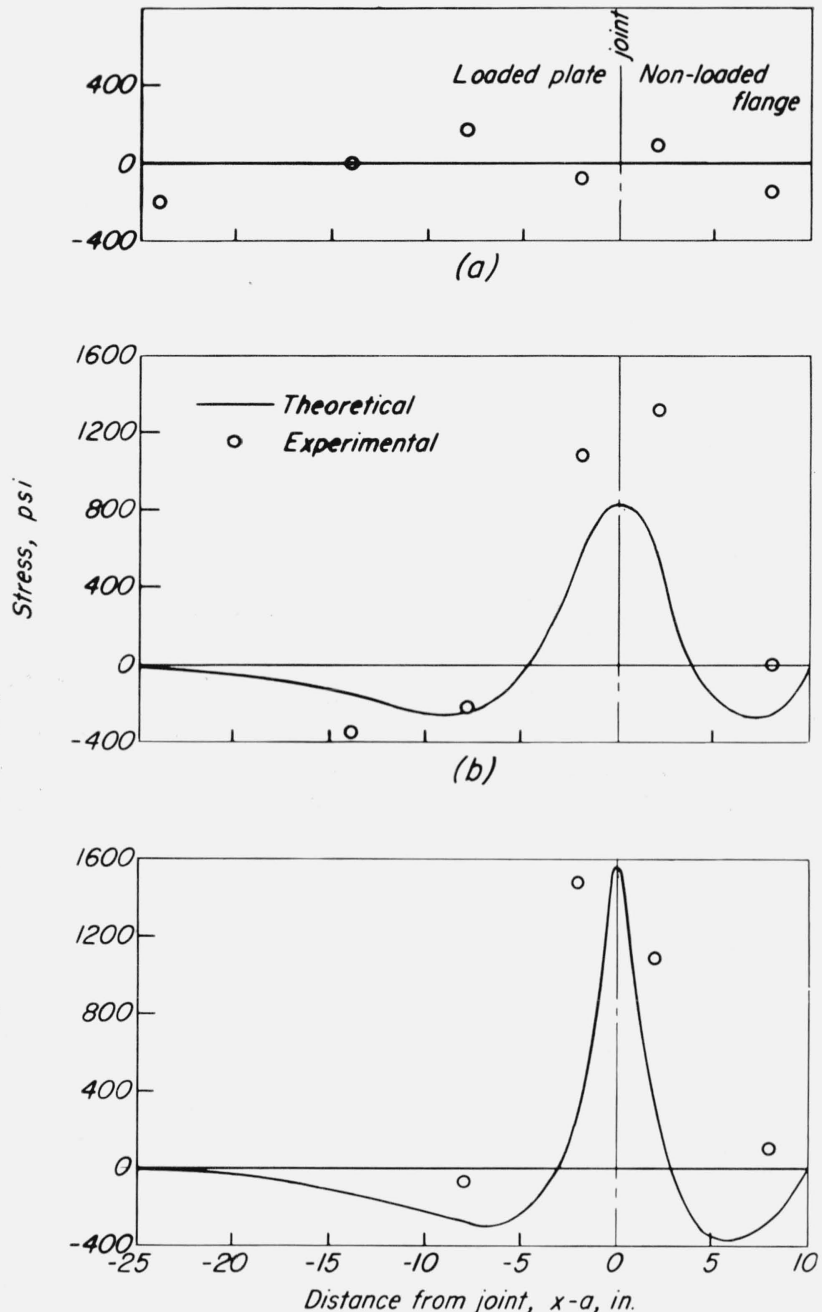

(c)

Figure 12. Shearing stresses in welded joint.

(a) Along center line; (b) 5 in. from center line; (c) 8 in. from center line.

\section{Application of Results to a Welded Structure}

Having computed the stresses in a plate loaded along portions of two opposite boundaries, an estimate of the stresses in a simple welded structure can be made. The unloaded flange is considered cut and attached to the end of the loaded base plate, figure 3. For a Poisson's ratio of 0.29 , the stresses corresponding to figure $4 \mathrm{~b}$ are

$$
\begin{gathered}
\sigma_{x}=p \\
\sigma_{y}=q=0.29 p .
\end{gathered}
$$

The stresses in a plate corresponding to figure $4 \mathrm{c}$ can be taken from tables 2 through 9 for appropriate geometrical parameters, noting that $(x-a)$ is the distance measured from the point of discontinuity of the load $q$. The stresses corresponding to those
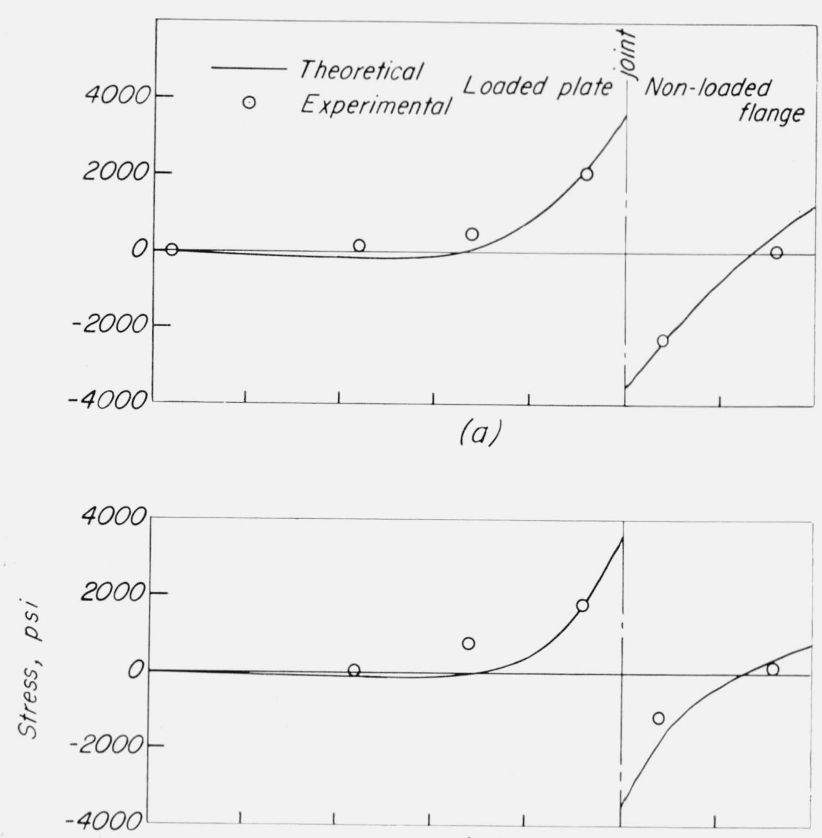

(b)

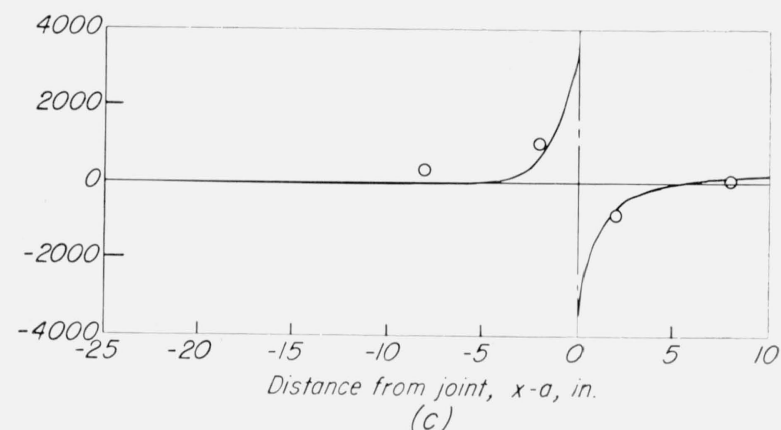

Figure 13. Stresses in transverse direction in welded joint.

(a) Along center line; (b) 5 in. from center line; (c) 8 in. from center line.

in figure $4 \mathrm{c}$ are then added to the stresses corresponding to those in figure $4 \mathrm{~b}$.

Using the tables as described above, the stress distribution in a welded structure similar to figure 2 was calculated. These theoretical results are given in figures 11 to 13 where they are compared with the experimental results described in the appendix. The results are found to be in reasonable agreement.

\section{Summary}

Values of the stresses in plates having uniform symmetrical loading along portions of two opposite longitudinal edges are presented in tables 2 to 9 . The discontinuity of loading affects the stress distribution in the plates for a distance to either side of the discontinuity equal to the width of the plate.

By suitable transformations, these results can be used to evaluate the stresses near the joint of two perpendicular plates subjected to uniaxial tension. 
The computed results show that a partial loading of the edges of the plate produces a biaxial stress condition. In any welded structure subjected to the loading considered, the state of stress would be biaxial tension.

Theoretical results obtained by the use of tables 2 to 9 show reasonable agreement with experimental results.

The authors are indebted to Ruth M. Woolley for her competent work on the preliminary phases of this problem so that it could be expeditiously programed for the computer, and to L. K. Irwin, A. F. Kirstein, and others in the Engineering Mechanics Section for their valuable suggestions and help, especially in the experimental phase of the problem.

\section{References}

[1] Campbell, William R., Stress studies of welded ship structure specimens, Welding J. 30, No. 2, 68s-77s (Feb. 1951).

[2] Campbell, William R., Irwin, L. K., and Duncan, R. C. Stress studies of bulkhead intersections for welded tankers, Welding J. 31, No. 2, 68s-76s (Feb. 1952).

[3] Parker, E. R., Brittle Behavior of Engineering Structures, John Wiley \& Sons, New York, p. 282 (1957).

[4] Filon, L. N. G., On an approximate solution for the bending of a beam of rectangular cross-section under any system of loads, with special reference to points of concentrated or discontinuous loading, Trans. Roy. Soc. (London) Series A 201, 63-155 (1903).

[5] Goodier, J. N., Compression of rectangular blocks, and the bending of beams by non-linear distributions of bending forces, J. Appl. Mechanics (Trans. A.S.M.E.) 54, No. 18, 173-183 (1932).

[6] Timoshenko, S. and Goodier, J. N., Theory of Elasticity, 2 d ed., McGraw-Hill Book Co., Inc., New York, pp. 46-51 (1951).

[7] Sokolnikoff, I. S. and Sokolnikoff, E. S., Higher Mathematics for Engineers and Physicists, 2d ed., MeGrawHill Book Company, Inc., New York (1941).

[8] Barton, M. V., The circular cylinder with a band of uniform pressure on a finite length of the surface, J. Appl. Mech. (Trans. A.S.M.E.) 8, A-97-A-104 (1941).

[9] Sechler, E. E., Elasticity in Engineering, John Wiley \& Sons, Inc., New York, p. 134 (1952).

[10] Timoshenko, S., The approximate solution of twodimensional problems in elasticity, Phil. Mag. Series 6, 4\%, No. 282, 1095-1104 (June 1924).

\section{Appendix}

In order to verify the theoretical results obtained, a test of a simple welded structure was conducted in a 1,150,000-lb capacity horizontal testing machine.

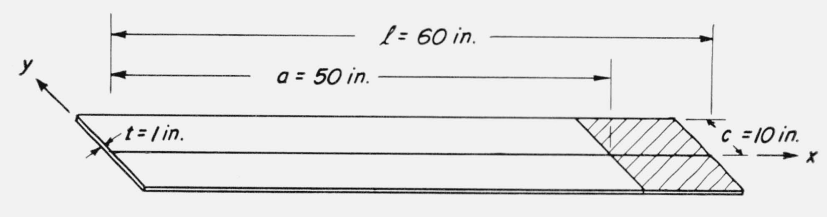

(a) Dimensions of welded structure.

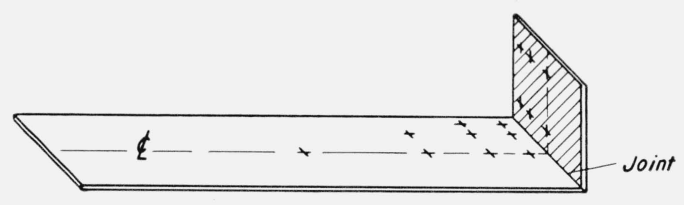

(b) Stations of which stresses were determined.

Figure 14. Welded structure tested.

The specimen was made of 40.8 -lb ship-plate, 1 in. in thickness. The base plate was $8 \mathrm{ft} .4$ in. long and $20 \mathrm{in}$. wide. Two flanges, each $20 \mathrm{in}$. wide and 10 in. long, were welded to the base plate (see fig. 14a). The overall shape of the specimen was similar to that shown in figure 2. Pulling heads having the shape of eyebars were welded to the ends of the base plate. The whole assembly was completely stressrelieved to a stress-free state. The pulling heads were then connected to the testing machine by pins. Bonded wire-resistance strain gages and threecomponent rosettes of bonded wire-resistance strain gages were installed at strategic locations on the specimen, figure $14 \mathrm{~b}$. With this setup it was possible to determine the Young's modulus and Poisson's ratio, as well as the magnitude of strains in different directions in the specimen. Poisson's ratio was determined to be 0.29 . The applied load was 500,000 $\mathrm{lb}$ or $p=25,000 \mathrm{lb} / \mathrm{in}$. in the longitudinal direction. The strains in the specimen were read directly to 10 $\mu \mathrm{in}$./in. and were estimated to $2 \mu \mathrm{in}$./in. Due to the high Young's modulus of steel, these values are equivalent to approximately 300 psi and $60 \mathrm{psi}$, respectively, in stresses. Therefore, the results cannot be claimed to have a high degree of accuracy.

The test results were reduced and indicated on the graphs of theoretical values (figs. 11 to 13), which are computed as described in section 8 . It can be seen that the theoretical and experimental results are in reasonable agreement.

(Paper 66C4-109) 